\title{
Optimal Rate Delay Tradeoffs and Delay Mitigating Codes for Multipath Routed and Network Coded Networks
}

\author{
John MacLaren Walsh, Member, IEEE, Steven Weber, Member, IEEE, Ciira wa Maina, Student Member, IEEE
}

\begin{abstract}
Via multiterminal information theory, a framework is presented for deriving fundamental rate delay tradeoffs that delay mitigating codes must have when utilized over multipath routed and random linear network coded networks. The rate delay tradeoff is formulated as a calculus problem on a capacity region of a related abstracted broadcast channel. Given this general framework for studying such rate delay tradeoffs, the extreme case of uniform networks, in which each possible received packet arrival order is equally likely, is considered. For these networks, the rate delay calculus problem is simplified to an integer programming problem, which for small numbers of packets may be solved explicitly, or for larger numbers of packets, may be accurately approximated through the calculus of variations by appropriate relaxation of an integer constraint. Explicit expressions for the rate delay tradeoff in uniform networks are presented in the special cases of $i$ ) constant packet inter-arrival times, and $i i$ ) exponential i.i.d. packet arrival times. Finally, the delay mitigating codes achieving these rate delay tradeoffs are discussed.
\end{abstract}

Index Terms-connection oriented service; delay mitigating codes; multipath routing; network coding; rate delay tradeoffs

\section{INTRODUCTION}

\section{A. Problem statement}

Consider a multipath routed network in which a particular source hands $M$ packets $\mathbf{x}_{1}, \ldots, \mathbf{x}_{M}$ (each $K$ bits long) to the lower network layers for transmission at time 0 . These $M$ packets are all headed to the same destination, but, because of queueing and propagation delays along the different routes, the packets $\left(\mathbf{x}_{1}, \ldots, \mathbf{x}_{M}\right)$ arrive at the sink at different time instants $\left(t_{1}, \ldots, t_{M}\right)$, possibly giving a different order. We will model the source to destination effects of the multi-path routed network transmission, then, as selecting these arrival times $t_{1}, \ldots, t_{M}$ randomly according to some joint distribution $\mathrm{p}\left(t_{1}, \ldots, t_{M}\right)$. We assume packets are not lost in order to focus our attention on the impact of packet reordering and delay (see $\S \mathrm{VI})$.

Denote by $\pi(i)$ the index of the transmitted packet that is the $i$ th received packet, so that the packets arriving at the receiver

Manuscript received April 9, 2008 and revised May 11, 2009. The work of J.M. Walsh and C. wa Maina was supported by NSF Grant 0728496.

All authors are with the Department of Electrical and Computer Engineering, Drexel University, Philadelphia, PA. Contact J. M. Walsh at jwalsh@ece.drexel.edu for information regarding this paper.

Preliminary versions of portions of this work were presented at the 2007 Allerton Conference on Communication, Control, and Computing [1] the 2008 Workshop on Network Coding, Theory, and Applications (NetCod) [2], and the 2008 International Symposium on Information Theory [3]. in order are $\mathbf{x}_{\pi(1)}, \mathbf{x}_{\pi(2)}, \ldots, \mathbf{x}_{\pi(M)}$, with ${ }^{1} t_{\pi(1)}<t_{\pi(2)}<$ $\ldots<t_{\pi(M)}$, which we denote by $\tau_{i}=t_{\pi(i)}$. We can then think of the probability distribution $\mathrm{p}\left(t_{1}, \ldots, t_{M}\right)$ on the arrival times as being composed of a probability distribution $\mathrm{p}(\pi)$ on the arrival order and a probability distribution on the ordered arrival instants given the arrival order $\mathrm{p}\left(\tau_{1}, \ldots, \tau_{M} \mid \pi\right)$. This paper investigates in particular the case where the ordered arrival instants are independent of the arrival order, so that $\mathrm{p}\left(\tau_{1}, \ldots, \tau_{M} \mid \pi\right)=\mathrm{p}\left(\tau_{1}, \ldots, \tau_{M}\right)$. Such independence occurs, for example, when the packet arrival times $t_{1}, \ldots, t_{M}$ are independent and identically distributed (i.i.d.).

Suppose further that the source node wishes to use the packets $\mathbf{x}_{1}, \ldots, \mathbf{x}_{M}$ to convey some temporally ordered data to the receiver. For instance, the source node may wish to convey a digital multimedia file that is the output of a multimedia source encoder which, after compressing the source, returns data organized into temporally ordered frames $\mathbf{s}_{1}, \ldots, \mathbf{s}_{N}$ corresponding to successive chunks of time from the multimedia signal. Assuming that the source encoder has done a good job of compressing, the data in $\mathbf{s}_{1}, \ldots, \mathbf{s}_{N}$ will be independent. Alternatively, the source node may be controlling the destination node remotely through the network, with a sequence of temporally ordered instruction frames $\mathbf{s}_{1}, \ldots, \mathbf{s}_{N}$ that must be executed in order. As another possibility, the source node may be engaged in a voice over IP (VoIP) conversation with the receiver. Indeed, almost any connectionoriented network source application could be considered as relevant for what we are about to discuss.

All of these temporally ordered source signals share the common characteristic that later data (i.e., with higher frame indices) is not useful until earlier data (i.e., with lower frame indices) has been received. However, since the effective point to point channel that the multi-path routed network creates reorders packets, were we simply to transmit the source data directly as is over the network channel, we would have to wait at the receiver, storing packets in a buffer, and reordering them as we played them out. Alternatively, one could consider a single source multicast network coded network employing random linear network coding [4], [5]. In this case $\left(\mathbf{x}_{1}, \ldots, \mathbf{x}_{M}\right)$ arriving at the sink will be random linear combinations of original transmitted packets (letting $M$ be the dimension of the global encoding vector), and we will have to wait, buffering the received packets, until all $M$ innovative (i.e., with linearly

\footnotetext{
${ }^{1}$ Since we are using continuous time, and thus continuously distributed arrival instants, the probability that two or more of the arrival instants are equal must be zero, so we ignore that case in our discussion.
} 
independent encoding vectors) packets have been received before we can start decoding even the earliest source frame $\mathbf{s}_{1}$. Here, we will model the innovative network encoded packets as arriving at times $t_{1}, \ldots, t_{M}$, giving again ordered arrival times $\tau_{1}<\cdots<\tau_{M}$.

While buffering has been extensively studied as a technique for connection oriented transmission over current networks such as the internet, we note that routing data from the same temporally ordered flow simultaneously along multiple paths and/or employing network coding for the sake of greater reliability and speed opens up a host of packet and information reordering phenomena. Buffering techniques designed for networks such as the present internet which generally maintain a FIFO (first in first out) delivery policy to the same packet flow [6], [7] may thus not be as appropriate for these situations. Thus, for these multipath routed and network coded networks we wish to consider an alternative to buffering: an approach based on coding. In particular, we propose to use a linear delay mitigating code to create the data in the transmitted packets from the source packets in such a way as to minimize the delay incurred while playing the source out at the receiver. This approach is to be contrasted with classical techniques that code for packet erasures with erasure correcting codes, where the focus is on delivering reliable transmission of a large block after a large delay. Our focus, instead, is to study the tradeoffs between rate and delay on these channels in a more concrete manner than allowed by, e.g., an error exponent based study.

\section{B. Solution techniques}

To achieve the goal of exhaustively characterizing and designing the best such delay mitigating codes, we use the following four step technique, depicted in Figure 1.

Step 1) Find the capacity region of the associated abstracted broadcast channel. Define an abstracted broadcast channel matching the packet re-ordering and combining characteristics of the network in question, in which different receivers correspond to the cumulative set of packet observations after different successive packet arrivals at the sink. Recognize this channel as a degraded broadcast channel, and calculate its capacity region $\mathcal{R}$, the general form of which is known [8], [9], [10]. This capacity region dictates the set of possible rate vectors $\mathbf{r}=\left(R_{1}, \ldots, R_{M}\right)$ over all possible delay mitigating codes. Here, each $R_{i}$ indicates the amount of new information that may be decoded upon each successive packet arrival at the sink (§III).

Step 2) Define the rate and delay. Define the overall rate $\rho(\mathbf{r})$ and overall delay $\delta(\mathbf{r})$ for a particular delay mitigating coding scheme in terms of the rate vector $\mathbf{r}$ (§IV-A). The overall rate will reflect the number of bits per second that will be delivered by the connection. The connection will provide this constant bitrate data in the form of a fixed number of new bits $\rho(\mathbf{r}) T_{s}$ delivered every $T_{s}$ seconds for $N T_{s}$ seconds. The chosen delay metric should reflect any deviation from this information delivery schedule.

Step 3) Determine the fundamental rate delay tradeoff. Find the optimal tradeoff between rate and delay by maximizing the overall rate $\rho(\mathbf{r})$ subject to a delay constraint $\delta(\mathbf{r}) \leq d$ over all feasible rate vectors $\mathbf{r}$ in the capacity region $\mathcal{R}$ :

$$
\rho_{d}^{*}:=\max _{\mathbf{r} \in \mathcal{R}}\{\rho(\mathbf{r}) \mid \delta(\mathbf{r}) \leq d\} .
$$

An optimal delay mitigating code is found as a capacity achieving code from the previous step with rate vector

$$
\mathbf{r}_{d}^{*} \in \arg \max _{\mathbf{r} \in \mathcal{R}}\{\rho(\mathbf{r}) \mid \delta(\mathbf{r}) \leq d\} .
$$

Our work also investigates a variational calculus approach for calculating in closed form the solutions to the rate delay tradeoff problem (1) in the limit of large numbers of packets. This amounts to approximating the rate vector, after appropriate scaling in the limit, as a continuous function mapping $[0,1]$ to $[0,1]$. The rate and delay are then normalized to each lie within $[0,1]$ and are approximated as functionals. In some cases this approximation can be aided by the theory of order statistics. The rate delay tradeoff programming problem then becomes a calculus of variations problem that we have solved in some special cases ( IV-C, $\S I V-D, \S I V-E)$.

Step 4) Determine capacity achieving codes for the abstracted degraded broadcast channel. These codes have rate vectors on the boundary of the capacity region $\mathcal{R}$. Despite the asymptotic block lengths employed in proving the degraded broadcast channel capacity region (which defeat the whole notion of bounding delay), surprisingly there exist codes block length of one (i.e., a single broadcast channel use) exhibiting the desired rate delay tradeoffs $(\S \mathrm{V})$ in the uniform case which is studied in this paper. In $\S \mathrm{V}$, capacity achieving codes in the uniform case are shown to be priority encoded transmission (PET) [11], [12] codes with an optimized collection of component code rates selected through the introduced framework. In other non-uniform cases, classes of codes other than PET codes are capacity achieving, but the rate delay tradeoffs we provide still hold as bounds.

\section{Overview of main results}

Section II discusses related work on error correction, fountain codes, priority encoded transmission, and error exponents. Section III gives capacity regions for two degraded broadcast channels modeling packet reordering with delays and network coded networks, respectively. Theorem 2 gives the rate region for the uniform permutation channel, i.e., when all packet reordering permutations are equally likely. Theorem 3 gives the analogous rate region for the uniform network coded channel, i.e., when the encoding matrix is selected uniformly at random over all matrices of given rank. In both cases the rate region is those vectors $\mathbf{r}=\left(R_{1}, \ldots, R_{M}\right)$ such that $\sum_{i=1}^{M} R_{i} / i \leq K$. Section IV presents the fundamental rate delay tradeoffs. While the presented rate delay tradeoff framework holds for more general situations, the remainder of the paper focusses on the uniform permutation and uniform network coded channel cases, which may be considered to be extreme cases of the more general problem. Theorem 4 gives the structural properties of the rate delay tradeoff in this uniform case, emphasizing that the problem can be parameterized in the decoding deadlines indicating the number of packets that must be received before decoding each frame. Because the decoding deadlines are integer valued this result transforms a 

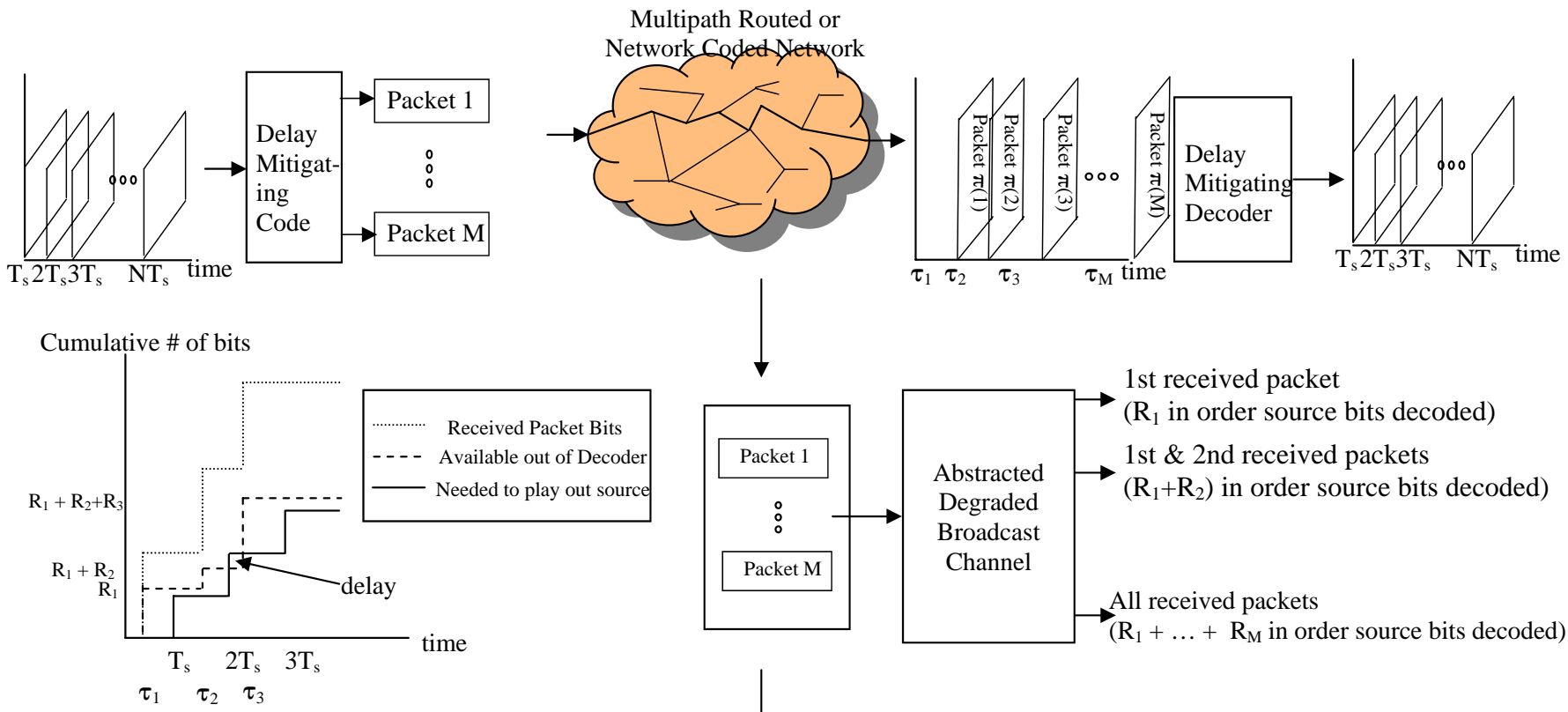

Capacity Region of Abstracted Broadcast Channel

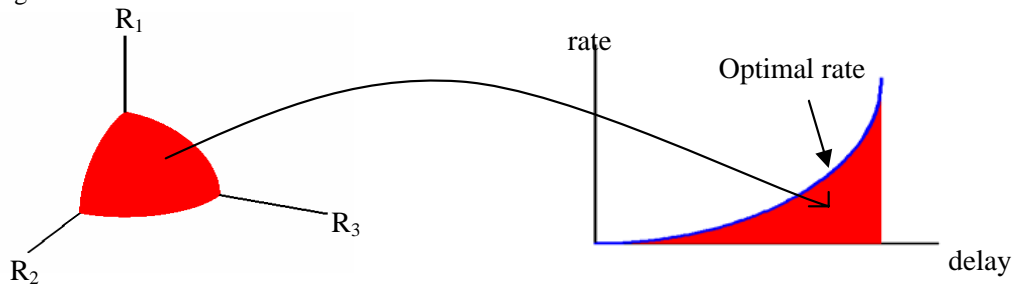

Fig. 1. The problem and the approach we will use to solve it.

continuous nonlinear program into an integer program, making the problem amenable to exact solution for small $M, N$. Then, Theorem 5 discusses a relaxation of the rate delay tradeoff problem via variational calculus. The integer-valued decoding deadline sequence is relaxed to a continuous function mapping $[0,1] \rightarrow[0,1]$. This relaxation gives a tractable solution which is a reasonable approximation of the optimal rate delay tradeoff for large $M, N$. Theorem 6 utilizes the approximation from the previous theorem to give an algebraic expression for the rate delay tradeoff in the special case of constant inter packet arrival times. Similarly, Theorem 7 gives an algebraic expression for the special case of i.i.d. exponential packet arrival times. In order to demonstrate the feasibility of the results, Section $\mathrm{V}$ discusses practical small blocklength codes achieving the rate delay tradeoffs. Section VI shows that although the rate delay tradeoffs are obtained using forward error correction (FEC), they are also optimal in a certain sense with respect to schemes incorporating feedback, e.g., TCP. Section VII contains a brief conclusion. Appendix A summarizes the key mathematical notation. Proofs of key theorems are presented in Appendices B through G.

\section{RELATED WORK}

Now that we have introduced the problem we wish to solve, and the technique we will use to solve it, we presently review some of the related literature.

\section{A. Erasure and Error Correction for Network Coded Networks}

One of the most important breakthroughs in the information theory community within the past decade has been the concept of network coding [4], [13], [14], [15] and its necessary utility in achieving the capacity of multi-cast networks. There is further a recent interest in erasure and error correction over network coded networks [16], [17], [18]. While some of this work can be used in delay mitigating codes research, e.g., [12], [19], it solves a different problem. The focus in network erasure and error correcting codes is typically designing block codes that can handle a particular (expected or exact) fraction of erasures or errors. Our work attempts to design codes which have unequal erasure protection (UEP), as in [12], [20], with a specific novel focus on choosing the UEP in such a way as to optimally trade transmission rate for delay. This builds into our model interest not just in the fraction (or expected fraction) of packets which are lost, but rather the way in which the channel combines, reorders, and delivers packets. Classic basic block erasure and error correcting codes do not incorporate these channel aspects.

Delay of random linear network coding is also addressed in [4], [21], where the delay properties of a particular random linear network coding scheme are assessed. Our scheme, in 
contrast, attempts to mitigate delay to be lower than that offered by unmodified random linear network coding by trading some of the rate with a concatenated (outer) delay mitigating code at the source. Alternatively, one could consider a fixed known network topology, and try to design the network code itself to accommodate both full rate transmission and zero delay, as was attempted in [22]. Designing such a network code requires global centralized coordination and therefore may not be feasible in practical networks. Our scheme, in contrast, is more likely to be feasible since it does not require any such global coordination.

\section{B. Fountain Codes}

Fountain codes [23], [24] are another related recent breakthrough in reliable broadcasting/multicasting over networks with users suffering different amounts of packet losses. Fountain codes, especially LT codes, can be seen as infinite block length (zero rate) analogs of irregular low density parity check (LDPC) codes [25], [26], [27], [28], [29], exploiting the same iterative low complexity belief propagation (BP) decoder, and the same sparse graph code construction. An unbounded number of packets are transmitted for a fixed block of source information. Their major innovation is that source information can be recovered with high probability and low computational complexity as long as a sufficient number of transmitted packets have been received by a particular receiver, no matter which subset of the original transmitted packets they were. Thus, different users can come in and out of the network, and can receive different subsets of transmitted packets, but as soon as they have received a number of bits that is $1+\epsilon$ times the number of source bits they can decode all of the source information with high probability.

Our delay mitigating codes are fundamentally different from fountain codes in a number of ways. First, our delay mitigating codes are not block codes at the decoder, unlike fountain codes which are. Since we are looking at a situation in which different information is decoded from all of the previously received packets upon each new packet arrival, block codes, and thus fountain codes do not apply. There have been some studies as to the fraction of bits that can be decoded in fountain codes before the necessary number of packets have been received to decode all of the source information [24], [30], [31]. Delay mitigating codes require not just that a certain fraction, say $k / n$, of the bits are recoverable, but in fact require the much stronger property that the recovered bits are the first $k$ of $n$ bits of the source. Even more, delay mitigating codes require a sequence of decoding deadlines be enforced, e.g., decode the first $k_{1}$ bits after receiving the first packet, decode the first $k_{1}+k_{2}$ packets after receiving the first two packets, etc. This requirement of enforcing a sequence of decoding constraints precludes the use of Raptor codes.

A second fundamental difference between delay mitigating codes and fountain codes, turbo codes [32], [33], [34], [35], or erasure correcting sparse graph codes, is that delay mitigating codes are for a different channel than these codes. The most common channel model for erasure correcting codes is the binary erasure channel (BEC) or packet erasure channel, which erases each bit (or packet) with a given probability $p$. Our codes, by contrast, are for an abstracted degraded broadcast channel relevant for trading rate for delay over multipath routed/network coded networks.

\section{Priority Encoded Transmission}

The above discussion of unequal error protection (UEP) brings up another body of older related work called PET codes, or codes for priority encoded transmission [11], [36] and their rank metric counterparts [12], [20]. The basic setup in PET and the related work [37], [38] involves a source comprised of different parts of information with different priorities, such as results from the use of a successive refinements encoder (e.g., as in the MPEG video standard [39], [40], [41]). Under successively decreasing prescribed erasure rates, source information of successively lower priority may be decoded. The PET construction is specified such that reception of any fraction $0<\rho_{i} \leq 1$ of the encoded packets guarantees that the data with priority greater than or equal to $i$ is all capable of being decoded $\left(\rho_{i}>\rho_{i-1}\right)$. To allow this, PET codes time share maximum distance separable (MDS) codes of different rates, and the time sharing construction can be thought as achieving points on a capacity region of an associated broadcast channel [36].

Our work is different from classic PET coding due to the focus on quantifying delay. In delay mitigating codes the different classes of source information correspond to different frames or time instants in the source data, with the requirement that earlier source data must be played out at the sink before later source data can be used. In a particular context of uniform network coded network and uniform multipath routed networks discussed later in the paper, our code construction amounts to optimally selecting the component rates $\rho_{i}$ for a PET code, and so PET codes are critical to our work. Selecting these component rates $\rho_{i}$ to correctly ensure specified delay constraints is in fact non-trivial and part of our work in this paper addresses this problem, see $\S \mathrm{V}$.

\section{Error Exponents as Delay Metrics}

A classic metric used as a surrogate for delay in information theoretic analysis of channels is the random coding error exponent [42]. The error exponent is the asymptotic exponential rate at which the sequence of error probabilities decays as a function of the block length in the limit of infinite block lengths when a random code is used. Because this metric is closely related to the necessary block length to achieve a certain bound on the probability of error for a given code rate, albeit when using a random code, it is often considered to be a reasonable metric related to the delay associated with the channel [43]. However, error exponents are inappropriate for our problem for two reasons.

First, the error exponents of codes we encounter in the context of this paper are infinite. In channels such as the $\mathrm{BEC}$, the error exponent is finite, reflecting the fact that the probability of error when using random codes, although decreasing to zero with increasing block lengths, is not zero for any finite block. The channels we are investigating, by 
contrast, do not share this characteristic. From the perspective of code design they are multi-terminal analogs of fixed erasure fraction channels, which have capacity achieving (MDS) codes with zero probability of error even for finite block lengths.

Second, the relationship between block length and temporal delay is tenuous in a network context. Rate measured in bits per channel use does not map smoothly to rate measured in bits per second when a channel use is defined as simultaneously transmitting a block of packets. This is because the packets take different amounts of time to traverse the network, owing to time spent waiting in different queues, and thus arrive at the sink for decoding at (unevenly spaced) random time instants. Using this channel repeatedly in a classic block code manner, the temporal rate at which information may be played out at the destination in bits per second is only indirectly related to the rate of the code in bits per channel use.

\section{Capacity Region of Some Relevant DegRaded BROADCAST CHANNELS}

In this section we introduce two abstracted broadcast channels in which different receivers correspond to successive packet arrivals at a common sink in the network. These two channels model the case of packets reordering with delays (§III-A) and network coded networks ( $(I I I-B)$, in a way that we will clarify in greater detail in (§III-C).

\section{A. The Permutation Channel Capacity Region}

Recall $\pi$ is the random permutation determining the order of packet arrivals at the destination, and $\mathrm{p}(\tilde{\pi})$ is the probability of each possible permutation $\tilde{\pi} \in \Pi$. We are interested in the region $\mathcal{R}$ of possible amounts of information that may be decoded upon each successive packet arrival at the sink. In particular, denote by $R_{j}$ the amount of new information that can be decoded after $j$ packets have arrived at the sink per collection of $M$ packets transmitted at a common time at the source. Collect these rates $R_{j}$ into a vector $\mathbf{r}=\left(R_{1}, \ldots, R_{M}\right)$. We can determine the region $\mathcal{R}$ of feasible (mutually satisfiable) rate vectors $\mathbf{r}$ as the capacity region of an abstracted degraded broadcast channel shown in Figure 2. Different receivers in the abstracted broadcast channel correspond to the cumulative packets received upon each successive packet reception at the sink. The packets are assumed to be labeled so that upon receiving the $i$ th packet, $\mathbf{x}_{\pi(j)}$, the destination knows both the payload $\mathbf{x}_{\pi(j)}$ and the packet index $\pi(j)$ (through the packet header). The observed values at the $j$ th receiver are $\mathbf{y}_{j}:=\left(\pi(1), \mathbf{x}_{\pi(1)}, \pi(2), \mathbf{x}_{\pi(2)}, \ldots, \pi(j), \mathbf{x}_{\pi(j)}\right)$. This broadcast channel is degraded; this is seen from the fact that the time reversed observations form a Markov chain through erasure operations $\mathbf{y}_{M} \rightarrow \mathbf{y}_{M-1} \rightarrow \cdots \rightarrow \mathbf{y}_{1}$.

Theorem 1 (Capacity Region of DBC [9], [8], [10], [44]): The capacity region of the degraded broadcast channel is the closure of the convex hull of the region $\mathcal{R}$ of rates satisfying

$$
\begin{aligned}
R_{1} & \leq \mathrm{I}\left(\mathbf{y}_{1} ; \mathbf{u}_{1}\right), \\
R_{2} & \leq \mathrm{I}\left(\mathbf{y}_{2} ; \mathbf{u}_{2} \mid \mathbf{u}_{1}\right), \ldots \\
R_{M} & \leq \mathrm{I}\left(\mathbf{y}_{M} ; \mathbf{x} \mid \mathbf{u}_{M-1}\right)
\end{aligned}
$$

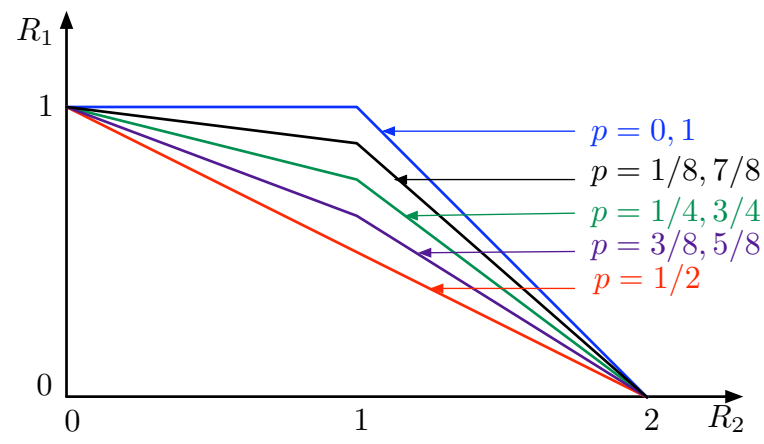

Fig. 3. The permutation channel's capacity region for $M=2$. The two extreme rate regions (known single permutation $(p \in\{0,1\})$ and uniform permutation selection $(p=1 / 2))$ are shown here, with $p$ indicating the probability that the packet numbered 1 when placed in the stack at the source node arrives first at the receiver. Note that the rate region boundaries for the intermediate cases lie in between those for these two extreme cases.

for a sequence of dummy discrete random variables $\mathbf{u}_{1} \rightarrow \cdots \rightarrow \mathbf{u}_{M-1} \rightarrow \mathbf{x}$ with bounded support, where $\mathbf{x}=\left(\mathbf{x}_{1}, \ldots, \mathbf{x}_{M}\right)$ is the channel input. ${ }^{2}$

Note that the auxiliary random variables in this theorem play the part of message variables in a random code [9], [8], [10], i.e., the decoder at receiver $j$ can determine the messages associated with the random variables $\mathbf{u}_{1}, \ldots, \mathbf{u}_{j}$, for each $j \in$ $\{1, \ldots, M\}$.

Proposition 1 (Permutation Channel Capacity Region): In the particular case of the permutation channel, the generic degraded broadcast channel capacity region expression can be simplified to

$$
\begin{aligned}
R_{1} & \leq \sum_{\tilde{\pi} \in \Pi} \mathrm{p}(\tilde{\pi})\left(\mathrm{H}\left(\mathbf{x}_{\tilde{\pi}(1)}\right)-\mathrm{H}\left(\mathbf{x}_{\tilde{\pi}(1)} \mid \mathbf{u}_{1}\right)\right), \ldots \\
R_{k} & \leq \sum_{\tilde{\pi} \in \Pi} \mathrm{p}(\tilde{\pi})\left(\mathrm{H}\left(\mathbf{x}_{\tilde{\pi}(1)}, \ldots, \mathbf{x}_{\tilde{\pi}(k)} \mid \mathbf{u}_{k-1}\right)\right. \\
R_{M} & \leq \mathrm{H}\left(\mathbf{x} \mid \mathbf{u}_{M-1}\right)
\end{aligned}
$$

The proof of this proposition is provided in Appendix B. From this expression we can view the capacity region as the image under a linear transformation whose coefficients are determined by the permutation distribution of the set of all possible entropy vectors created from joint entropies of subsets of the dummy random variables $\mathbf{u}_{1}, \ldots, \mathbf{u}_{M-1}, \mathbf{x}$. In the two special cases of interest we presently discuss, the expression for the rate region can be simplified even further.

Proposition 2 (Special Case: Single Permutation): When only one permutation is possible the amount of information received upon the $j$ th packet arrival is limited by the cumulative number of received bits. In particular, the rate region $\mathcal{R}$ is the collection $\mathbf{r}=\left(R_{1}, \ldots, R_{M}\right), \mathbf{r} \geq \mathbf{0}$

\footnotetext{
${ }^{2}$ The references [9], [8], [10], [44] mostly focus on the 2 user case, however there is no difficulty in extending the coding scheme [10] and converse proofs [44] to the presented $M$ user case.
} 


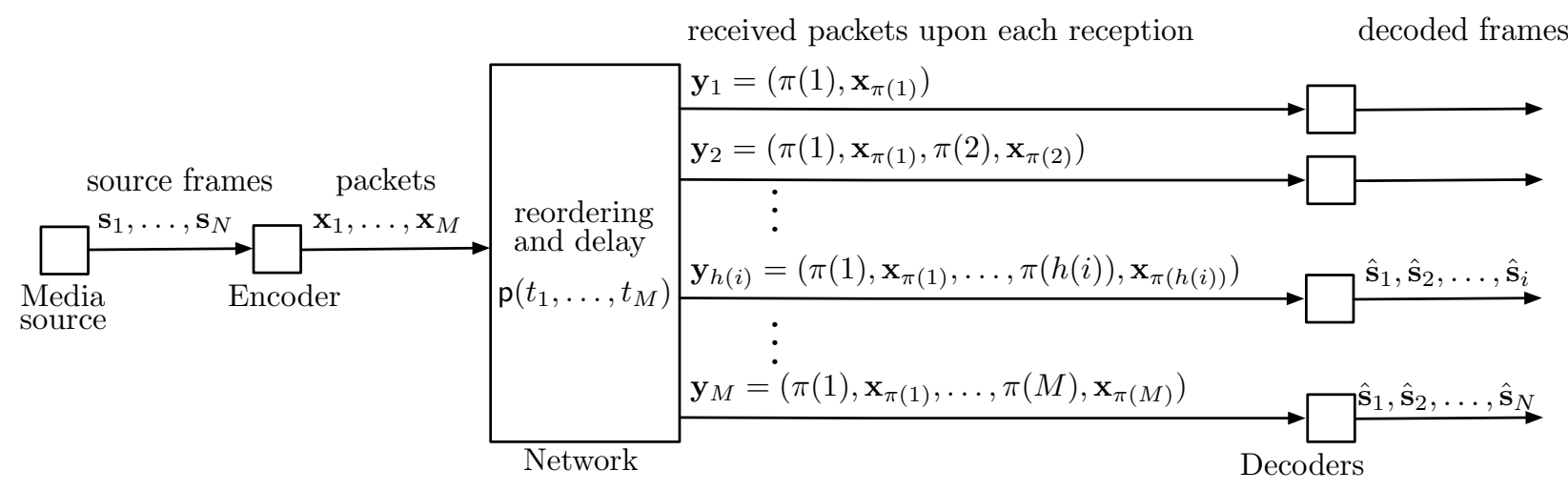

Fig. 2. The permutation channel reinterpreted as an abstracted degraded broadcast channel for the purpose of analyzing rate delay tradeoffs. Upon reception of the first $h(i)$ packets the sink may decode the first $i$ frames. The decoding deadlines $\mathbf{h}=(h(1), \ldots, h(N))$ are illustrated in Figure 7.

specified by the $M$ inequalities

$$
\sum_{j=1}^{m} R_{j} \leq m K, \quad \forall m \in\{1, \ldots, M\} .
$$

This rate region for $M=2$ is shown as the $p \in\{0,1\}$ region in Figure 3. An additional interesting special case occurs when the distribution on the reordering permutation $\tilde{\pi}$ is uniform. Such a uniform distribution for $\pi$ occurs, for instance, via symmetry when the unordered arrival times $\left\{t_{j}\right\}$ are independently and identically distributed.

Theorem 2 (Uniform Permutation Channel): When all permutations are equally likely $(\mathrm{p}(\tilde{\pi})=1 /|\Pi|$ for each $\tilde{\pi} \in \Pi)$ the capacity region is specified by the inequality:

$$
\mathcal{R}=\left\{\mathbf{r}=\left(R_{1}, \ldots, R_{M}\right): \mathbf{r} \geq \mathbf{0}, \sum_{j=1}^{M} \frac{R_{j}}{j} \leq K\right\} .
$$

The proof is found in Appendix C. This rate region for $M=2$ is shown as the $p=1 / 2$ region in Figure 3 .

\section{B. Network Coded Case}

In the random linear network coded case where the packets that arrive at time instants $\left\{\tau_{j}\right\}$ are linear combinations of the transmitted packets, we are again interested in a similar abstracted degraded broadcast channel (DBC), depicted in Figure 4. The different receivers correspond to successive innovative packet arrivals and the vectors $\mathbf{g}_{k}$ correspond to the global encoding vector of the $k$ th received packet. Because we are considering random linear network coding from a source to sink viewpoint, we model a random linear network coded network as a device that selects the random global encoding matrices $\mathbf{G}_{k}=\left[\mathbf{g}_{1}^{\top}, \mathbf{g}_{2}^{\top}, \ldots, \mathbf{g}_{k}^{\top}\right]^{T}$ according to a probability distribution $\mathrm{p}\left(\widetilde{\mathbf{G}}_{k}\right)$ on $\mathcal{G}_{k}$ the set of $k \times M$ matrices with rank $k$. The rate region corresponding to the amounts of new information that can be decoded upon successive innovative packet arrivals at the sink is given by

$$
\begin{aligned}
R_{1} & \leq \sum_{\tilde{\mathbf{G}}_{1} \in \mathcal{G}_{1}} \mathrm{p}\left(\tilde{\mathbf{G}}_{1}\right)\left(\mathrm{H}\left(\tilde{\mathbf{G}}_{1} \mathbf{x}\right)-\mathrm{H}\left(\tilde{\mathbf{G}}_{1} \mathbf{x} \mid \mathbf{u}_{1}\right)\right) \\
R_{k} & \leq \sum_{\tilde{\mathbf{G}}_{k} \in \mathcal{G}_{k}} \mathrm{p}\left(\tilde{\mathbf{G}}_{k}\right)\left(\mathrm{H}\left(\tilde{\mathbf{G}}_{k} \mathbf{x} \mid \mathbf{u}_{k-1}\right)-\mathrm{H}\left(\tilde{\mathbf{G}}_{k} \mathbf{x} \mid \mathbf{u}_{k}\right)\right) \\
R_{M} & \leq \mathbf{H}\left(\mathbf{x} \mid \mathbf{u}_{M-1}\right)
\end{aligned}
$$

where (11) ranges over $k \in\{2, \ldots, M-1\}$. This region follows from substituting the definition for the $M$ received signals in the network coded context into (3), (4), and (5), and mimicking the steps detailed in Appendix B.

We consider next the specific case where we can model the source to destination channel created by network coding as sampling the random encoding matrices $\mathbf{G}_{k}$ uniformly from the set $\mathcal{G}_{k}$ of all encoding matrices in $\operatorname{GF}(q)^{k \times M}$ of rank $k$ (henceforth the uniform network coded channel). In this instance, the capacity region of the abstracted broadcast channel determining the amounts of information decodable upon each successive packet arrival is given by the following theorem.

Theorem 3 (Uniform Network Coded Channel Capacity Region): For the uniform network coded channel $(p(\tilde{\mathbf{G}})$ is uniform) the capacity region is specified by the inequality:

$$
\mathcal{R}=\left\{\mathbf{r}=\left(R_{1}, \ldots, R_{M}\right): \mathbf{r} \geq \mathbf{0}, \sum_{j=1}^{M} \frac{R_{j}}{j} \leq K\right\} .
$$

The proof is found in Appendix D. Note that this rate region is the same as obtained for the uniform packet reordering channel in Theorem 2.

\section{Relating the Abstracted Broadcast Channel to Delay Mit- igating Codes}

Having introduced the two abstracted broadcast channels (Theorems 2 and 3), we now discuss capacity achieving codes for these channels, their associated fundamental limits, and their connection with the problem of connection oriented transmission for multi-path routed and network coded networks. 


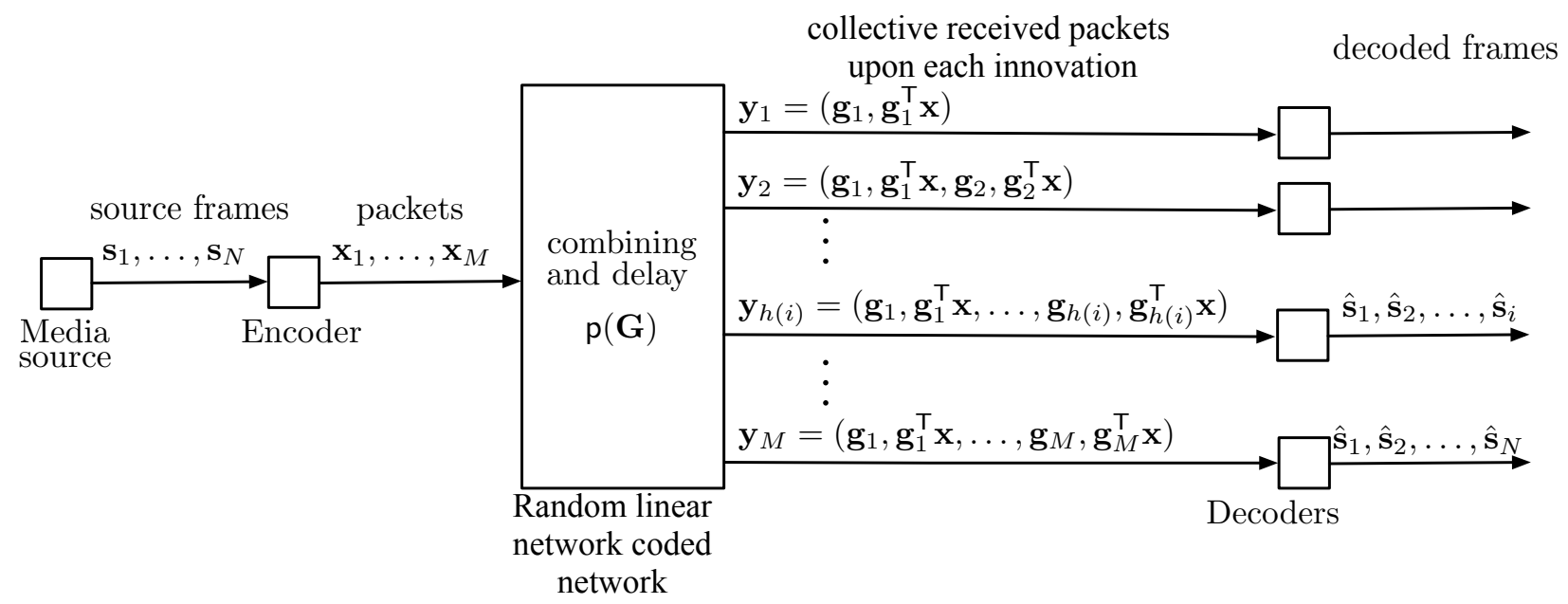

Fig. 4. Point to point network coded channel as a degraded broadcast channel.

First, it is important to point out that Figures 2 and 4 detail a single use of the discrete memoryless channel under consideration. A block code with block length $L$ for this discrete memoryless channel would have codewords which individually are a block of $L$ collections of $M$ packets ( $L M$ packets all together), labeled $\mathbf{x}^{1}, \ldots, \mathbf{x}^{l}, \ldots, \mathbf{x}^{L}$ with $\mathbf{x}^{l}:=\left[\mathbf{x}_{1}^{l}, \ldots, \mathbf{x}_{M}^{l}\right]$. For the permutation channel, the $j$ th receiver receives a sequence of $L$ collections of $j$ packets:

$$
\mathbf{y}_{j}^{L}=\left[\left(\pi_{l}(1), \mathbf{x}_{\pi_{l}(1)}^{l}, \ldots, \pi_{l}(j), \mathbf{x}_{\pi_{l}(j)}^{l}\right) \mid l \in\{1, \ldots, L\}\right] .
$$

Due to the memoryless nature of the channel there are $L$ independent and identically distributed permutations governing the arrival order of each of the $L$ collections of $M$ packets $\pi_{1}:=\left(\pi_{1}(1), \ldots, \pi_{1}(M)\right), \pi_{2}:=\left(\pi_{2}(1), \ldots, \pi_{2}(M)\right)$, through $\pi_{L}:=\left(\pi_{L}(1), \ldots, \pi_{L}(M)\right)$. Because this is clearly a discrete memoryless degraded broadcast channel, the capacity region theorem can be applied, and a capacity region $\mathcal{R}$ of achievable rate vectors $\mathbf{r}=\left(R_{1}, \ldots, R_{M}\right)$ with $R_{j}$ signifying the number of bits deliverable per channel use to receiver $j$. The collective amount of error-free information decoded by receiver $j$ will be $L R_{j}$. Similarly for the network coded channel, Figure 4 details a single use of the associated discrete broadcast channel, while a block code would code over multiple such channel uses.

Second, we emphasize that the capacity regions in Theorems 2 and 3 hold under an assumed uniform distribution: equally likely packet arrival orders (permutations) for Theorem 2 and equally likely encoding matrices of each rank for Theorem 3 . A more general problem is to determine the capacity of the channels under an arbitrary distribution on permutations and encoding matrices, respectively, but still assuming the ordered arrival instants $\tau_{j}:=t_{\pi(j)}, j \in\{1, \ldots, M\}$ are independent of the arrival order $\pi$. An even more general problem is to relax this assumption and allow dependence between the arrival order and the ordered arrival times. A uniform distribution for $\pi$ and independence between $\tau$ and $\pi$ happen, for instance, if the unordered arrival times, $t_{1}, \ldots, t_{M}$, are independently and identically distributed. This assumption is valid in a network with $M$ independent paths through the network where each path has an identical delay distribution. Naturally, we would like to understand with some level of rigor this statistically symmetric case before delving into the more complex, yet more realistic, irregular cases.

We want to use a multipath network like this to provide a constant bit rate connection between the source and destination, in the sense of providing a new frame containing a constant number of bits every $T_{s}$ seconds, and we want these frames to be played out in a specified order without any being omitted. The reordering phenomenon caused by the random arrival order makes this difficult to do without introducing extra delay if uncoded transmission is to be used, because we must buffer the received packets so we can play them out in order. Alternatively we can encode the contents of the $M$ transmitted packets. This would correspond to a block code (as discussed above) for the introduced abstracted degraded broadcast channel with block length $L=1$. The number of bits $R_{j}$ that can be guaranteed (with probability 1 ) to be delivered by this block code after $j$ packets have been received must yield a rate vector $\mathbf{r}=\left(R_{1}, \ldots, R_{M}\right)$ lying inside the capacity region $\mathcal{R}$ for the associated degraded broadcast channel. To see this (in spite of the infinite block lengths used in the typical degraded broadcast channel capacity region proof), simply note we can make a block length $L$ code with rate (per channel use) vector $\mathbf{r}$, by concatenating $L$ of our block length 1 codes end to end. Since we are only considering error free block length 1 codes, this code can not be any better than the fundamental limits for reliable communication as $L \rightarrow \infty$ provided by the degraded discrete memoryless broadcast channel capacity theorems. For many discrete memoryless degraded broadcast channels, not all rate vectors $\mathbf{r}$ may be achieved with a block length $L=1$, and thus $\mathcal{R}$ forms a strict outer bound for the set of block length $L=1$ code rate vectors that we are interested in. In these cases, the calculus on the associated rate region to trade aggregate bit rate for aggregate delay that we discuss in the next section would yield bounds for the associated rate delay tradeoff which could not be reached with practical codes. However, as we shall show in $\S \mathrm{V}$, the channel we are studying has nontrivial 
practical codes which achieve points on the boundary of the capacity region with block length $L=1$, and thus the rate delay tradeoffs we present are achievable with practical and low-complexity decodable codes.

\section{Fundamental Rate Delay Tradeoffs}

In this section the capacity regions obtained in $\S I I I$ are used to describe fundamental rate delay tradeoffs in uniform multipath routed and network coded networks. Rate and delay are defined in $\S \mathrm{IV}$-A relative to a constant bit rate source, along with the optimization problem whose solution is the rate delay tradeoff. This section ( $(\mathrm{IV}-\mathrm{A})$ is general, and can be applied out of the scope of the limited uniform multipath routed and network coded cases. In $\S$ IV-B we identify the structural properties of this optimization problem in the uniform cases, showing that it may be simplified to an integer programming problem, amenable to exact exhaustive solution for small $M, N$. In order to make this problem more tractable for large $M, N, \S$ IV-C introduces a variational relaxation of the integer programming problem. Algebraic expressions for the rate delay tradeoff are obtained by solving this variational calculus problem for some special packet arrival time statistics in $\S I V-D$ (constant inter arrival times) and $\S I V-E$ (exponential i.i.d. arrival times).

\section{A. Rate Delay Calculus Problem Formulation}

Theorems 2 and 3 give the region $\mathcal{R}$ describing the amounts of new information available after each successive packet arrival at the sink, provided the permutations and encoding matrices, respectively, are selected uniformly. We can incorporate the inter-arrival statistics with these results to determine the information transmission rate (in bits per second) and delay (in seconds) associated with each point $\mathbf{r}$ in the capacity region. With the connection oriented context in mind, suppose that the source decoder at the sink node needs to decode a new frame $\mathbf{s}_{i}$ every $T_{s}$ seconds. Then the overall rate at which data is decoded at the source decoder at the sink is

$$
\rho(\mathbf{r}):=\frac{1}{N T_{s}} \sum_{j=1}^{M} R_{j}
$$

bits per second. This means we are trying to decode $\frac{1}{N} \sum_{j=1}^{M} R_{j}$ bits every $T_{s}$ seconds.

We next define a class of delay metrics, parameterized by $p \in[1, \infty)$, for each rate vector $\mathbf{r}$ that penalizes the (random) delay incurred by every frame, according to the expression

$$
D_{p}(\mathbf{r})=\sum_{i=1}^{N}\left[\left(\tau_{g(i, \mathbf{r})}-i T_{s}\right)^{+}\right]^{p}
$$

where $(x)^{+}:=\max \{x, 0\}$ and

$$
g(i, \mathbf{r}):=\inf \left\{n \mid \sum_{j=1}^{n} R_{j} \geq i T_{s} \rho(\mathbf{r})\right\} .
$$

Here, $g(i, \mathbf{r})$ is the number of packets that must be received before the sink can decode the first $i$ frames of the source. The delay is a random variable as it is a function of the arrival instants $\left\{\tau_{j}\right\}$. Note that the assumed functional form (14) allows a wide variety of delay metrics, reflecting the fact that the overall delay will be likely to be a function of the delay $\left(\tau_{g(i, \mathbf{r})}-i T_{s}\right)^{+}$of $i$ th frame, which is the difference between the time $i T_{s}$ it is to be played out, and the (random) time it is guaranteed to be available $\tau_{g(i, \mathbf{r})}$. Due to properties of the $p$ norms, the family of functionals (14) can thus be stretched all the way from being proportional to the sum of the frame delays, to being proportional to the worst frame delay by using larger and larger powers $p$.

At the heart of the idea of rate delay calculus is the intent of finding the point $\mathbf{r}$ in the coding achievable rate region $\mathcal{R}$ which maximizes the overall rate $\rho(\mathbf{r}) \leq \rho_{d}^{*}$ among all codes with expected delay $\delta(\mathbf{r})=\mathbb{E}\left[D_{p}(\mathbf{r})\right]$ less than or equal to some bound $d$. The optimal rate delay tradeoff is

$$
\rho_{d}^{*}:=\max _{\mathbf{r} \in \mathcal{R}}\left\{\rho(\mathbf{r}) \mid \mathbb{E}\left[D_{p}(\mathbf{r})\right] \leq d\right\} .
$$

\section{B. Structural Properties of the Rate Delay Tradeoff in Uniform Case}

Because the rate regions in Theorems 2 and 3 are the same, calculating the rate delay tradeoffs for these two channels amounts to the same calculus problem. Thus, for the remainder of this article, we handle these two cases simultaneously, focussing entirely on the uniform cases. The next theorem shows that in these uniform cases, the rate delay tradeoff, although correctly posed as a continuous optimization, is found as the solution to an integer programming problem (with a finite set of feasible solutions).

Theorem 4 (Structural Properties of Rate Delay Tradeoff): When the channel selects the permutation uniformly from the set of all permutations, the optimal rate delay tradeoff (16) may be obtained by solving the optimization problem

$\rho_{d}^{*}=\max _{\mathbf{h}}\left\{\rho(\mathbf{h})=\frac{K}{T_{s}}\left(\sum_{m=1}^{n(\mathbf{h})} \frac{\Delta(\mathbf{h})_{m}}{l(\mathbf{h})_{m}}\right)^{-1} \mid \mathbb{E}\left[D_{p}(\mathbf{h})\right] \leq d\right\}$

over the decoding deadlines $\mathbf{h}=(h(i), i=1, \ldots, N)$ which are nondecreasing integer valued functions mapping $\{1, \ldots, N\} \rightarrow\{1, \ldots, M\}$. Here $h(i)$ indicates the number of packets that must be received before decoding the first $i$ frames, $n(\mathbf{h})$ is the number of distinct values of $\mathbf{h}, l(\mathbf{h})_{m}$ is the $m$ th distinct value of $\mathbf{h}$, and $\Delta(\mathbf{h})_{m}$ is the number of frame indices associated with the $m$ th distinct level of $\mathbf{h}$. Finally, $D_{p}(\mathbf{h})$ equals $D_{p}(\mathbf{r})$ as defined in (14) but with $g(i, \mathbf{r})=h(i)$ in (15) for each $i=1, \ldots, N$.

The proof is found in Appendix E. This structural transformation to an integer programming problem over $\mathbf{h}$ allows direct calculation of the rate delay tradeoffs by explicit enumeration over all nondecreasing sequences satisfying the delay bound.

\section{Variational Approximation based Rate Delay Tradeoff for} Large $M, N$

For large $M, N$ the solution by explicit enumeration in Theorem 4 is computationally infeasible, necessitating an 
approximate solution. We obtain an approximation by transforming the integer programming problem into a variational calculus problem over a family of non-decreasing continuous functions, whose solution, via Lagrangian techniques, can be found as a solution to an algebraic equation. In order to avoid unbounded rates and delays as $M, N$ grow large, we first normalize the rate and delay metrics to lie in between 0 and 1.

Since $\sum_{j=1}^{M} R_{j} \leq M K$ it follows that $\rho(\mathbf{r})$ in (13) and hence $\rho(\mathbf{h})$ in (17) is upper bounded by $\frac{M K}{N T_{s}}$. The normalized rate is then

$$
\bar{\rho}(\mathbf{h})=\frac{N T_{s}}{M K} \rho(\mathbf{h})=\frac{N}{M}\left(\sum_{m=1}^{n(\mathbf{h})} \frac{\Delta(\mathbf{h})_{m}}{l(\mathbf{h})_{m}}\right)^{-1} .
$$

The normalized delay is obtained by noting that our delay metric is monotone non-decreasing in $h(i)$, and thus the greatest delay is obtained when the decoding deadlines are $h(i)=M$ for each $i=1, \ldots, N$. Thus, to obtain a normalized delay lying in between 0 and 1 , we need only to divide the expected non-normalized random delay metric by its expected value at the extreme decoding deadlines:

$$
\bar{\delta}(\mathbf{h}):=\frac{\mathbb{E}\left[D_{p}(\mathbf{h})\right]}{\mathbb{E}\left[D_{p}(\{h(i)=M, \forall i \in\{1, \ldots, N\}\})\right]} .
$$

In order to approximate the solution to the integer programming problem by the solution to a variational calculus problem, first define a zero order hold piecewise continuous function $\mathrm{z}:[0,1] \rightarrow[0,1]$ such that $\mathrm{z}\left(\frac{i}{N}\right)=\frac{h(i)}{M}:$

$$
\mathbf{z}(t)=\sum_{i=1}^{N} \frac{h(i)}{M} \mathbf{1}_{\frac{i-1}{N} \leq t<\frac{i}{N}}, t \in[0,1] .
$$

The normalized rate $\rho(\mathbf{h})$ may be expressed in terms of $z$ as the functional $\bar{\rho}[\mathrm{z}]$ according to

$$
\bar{\rho}(\mathbf{h})^{-1}=\sum_{m=1}^{n(\mathbf{h})} \frac{1}{\frac{l(\mathbf{h})_{m}}{M}} \frac{\Delta(\mathbf{h})_{m}}{N}=\bar{\rho}[\mathbf{z}]^{-1}=\int_{0}^{1} \frac{1}{\mathbf{z}(t)} \mathrm{d} t .
$$

The normalized delay is then approximated by a functional $\bar{\delta}[z]$ of $z$ as well:

$$
\begin{aligned}
\bar{\delta}[\mathbf{z}] & =\frac{\frac{1}{N} \sum_{i=1}^{N} \mathbb{E}\left[\left[\left(\tau_{M z(i / N)}-\frac{i}{N} N T_{s}\right)^{+}\right]^{p}\right]}{\frac{1}{N} \sum_{i=1}^{N} \mathbb{E}\left[\left[\left(\tau_{M}-\frac{i}{N} N T_{s}\right)^{+}\right]^{p}\right]} \\
& \rightarrow \frac{\int_{0}^{1} \mathbb{E}\left[\left[\left(\tau_{M z(t)}-t N T_{s}\right)^{+}\right]^{p}\right] \mathrm{d} t}{\int_{0}^{1} \mathbb{E}\left[\left[\left(\tau_{M}-t N T_{s}\right)^{+}\right]^{p}\right] \mathrm{d} t},
\end{aligned}
$$

for $N$ large.

The rate delay tradeoff for large $M$ is obtained by solving the calculus of variations [45], [46] problem for a normalized delay bound $\bar{d} \in[0,1]$ :

$$
\bar{\rho}_{\bar{d}}^{*}:=\max _{\mathrm{z}:[0,1] \rightarrow[0,1]}\left\{\bar{\rho}[\mathrm{z}] \mid \bar{\delta}[\mathrm{z}] \leq \bar{d}, \quad \frac{\partial \mathrm{z}(t)}{\partial t} \geq 0\right\} .
$$

Here, $\mathrm{z}$ is no longer constrained to be a piecewise constant function, and, in fact, is now considered among the class of continuously differentiable non-decreasing functions. For large $M$ (where explicit enumeration of $\mathbf{h}$ is too difficult) the intervals on which $\mathrm{z}$ was to be piecewise constant should be very small, so one would expect to get a good approximation for these cases. Using monotonicity of the function $\frac{1}{x}$, the problem (20) may be cast in standard variational calculus form:

$$
\frac{1}{\bar{\rho}_{\bar{d}}^{*}}:=\min _{\mathrm{z}:[0,1] \rightarrow[0,1]}\left\{\int_{0}^{1} \frac{1}{\mathrm{z}(t)} \mathrm{d} t \mid \bar{\delta}[\mathrm{z}] \leq \bar{d}, \frac{\partial \mathrm{z}(t)}{\partial t} \geq 0\right\} .
$$

The following theorem gives a general form for the optimal decoding deadline function $z$ under an even more general family of delay metrics than (19).

Theorem 5 (Variational Approximation to Rate Delay Tradeoff): Suppose the normalized delay function may be written as an integral of a function $\mathrm{Q}(t, \mathrm{z}(t))$, i.e.,

$$
\bar{\delta}[\mathbf{z}]=\int_{0}^{1} \mathrm{Q}(t, \mathrm{z}(t)) \mathrm{d} t .
$$

Then for those $t_{0}$ such that $\left.\frac{\partial z^{*}}{\partial t}\right|_{t=t_{0}} \neq 0$, the optimal decoding deadline function $z_{\bar{d}}^{*}$ to solve (20) is the solution $z$ to the equation

$$
\mathbf{z}\left(t_{0}\right)=\left(\mu \frac{\partial \mathrm{Q}\left(t_{0}, \mathbf{z}\left(t_{0}\right)\right)}{\partial \mathbf{z}\left(t_{0}\right)}\right)^{-\frac{1}{2}},
$$

with $\mu=\mu_{\bar{d}}$ selected so that $\bar{\delta}\left[\mathrm{z}_{\bar{d}}^{*}\right] \leq \bar{d}$. The rate delay tradeoff is then given by $\bar{\rho}_{\bar{d}}^{*}=\bar{\rho}\left[\mathbf{z}_{\bar{d}}^{*}\right]$.

Proof The Lagrangian $\mathcal{L}(z, \mu, a)$ is

$$
\int_{0}^{1} \frac{1}{\mathrm{z}(t)} \mathrm{d} t+\mu\left(\int_{0}^{1} \mathrm{Q}(t, \mathrm{z}(t)) \mathrm{d} t-\bar{d}\right)+\int_{0}^{1} \mathrm{a}(t) \frac{\partial \mathrm{z}(t)}{\partial t} \mathrm{~d} t,
$$

where $\mathrm{a}(t)$ is the Lagrange multiplier on the constraint $\frac{\partial z(t)}{\partial t} \geq$ 0 at $t$, for each $t \in[0,1]$. The solution to the variational problem (20) must satisfy the algebraic equation obtained by differentiating $\mathcal{L}(\mathrm{z}, \mu, \mathrm{a})$ at each $t=t_{0}$ :

$$
-\frac{1}{\left(\mathbf{z}^{*}\left(t_{0}\right)\right)^{2}}+\left.\mu \frac{\partial \mathrm{Q}\left(t_{0}, x\right)}{\partial x}\right|_{x=\mathbf{z}^{*}\left(t_{0}\right)}+\alpha\left(t_{0}\right) \mathbf{1}_{\left\{\frac{\partial z^{*}\left(t_{0}\right)}{\partial t}\right\}}=0
$$

for each $t_{0} \in[0,1]$, with $\mu, \mathrm{z}^{*}(0)$ such that $\bar{\delta}\left[\mathrm{z}^{*}\right] \leq \bar{d}$ and $\alpha(t)$ selected so as to make $z^{*}$ constant on intervals where it is constant (and thus it is not necessary to actually solve for it).

Note the family of functionals (19) is a particularly useful form of the more general class of functionals (22). We next specialize Theorem 5 to the special cases of constant packet inter-arrival times ( $(I V-D)$ and i.i.d. packet inter-arrival times (§IV-E).

\section{Rate Delay Tradeoffs for Constant Packet Inter-arrival Times}

Consider the case when $\tau_{j}=j T_{s}$ and $N=M$, so that time is slotted on packet arrivals and the only remaining random 


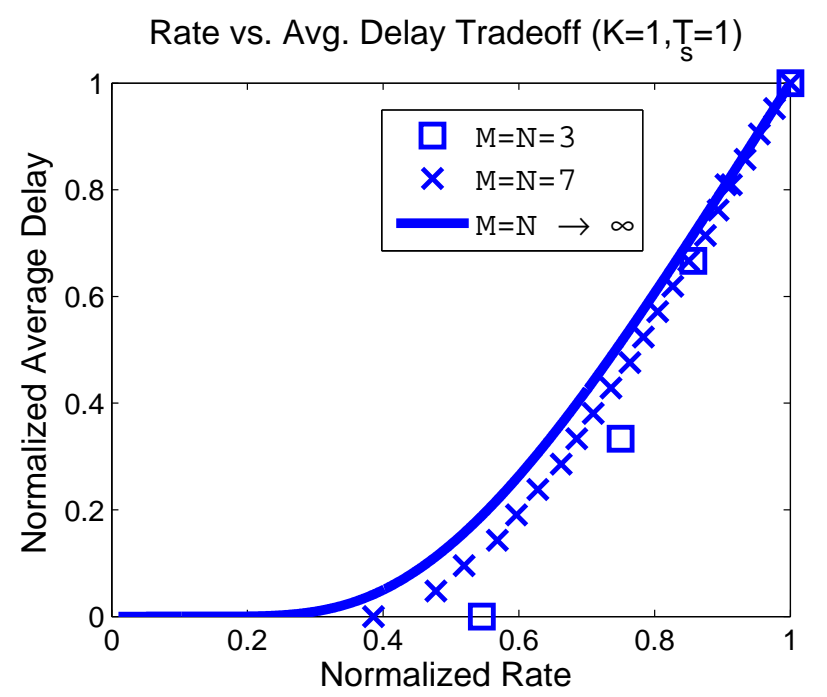

Fig. 5. Exact and approximate rate delay tradeoffs when the (reordered) packets arrive in a constant periodic manner.

element in the system is the uniform permutation selection. The normalized delay functional becomes

$$
\begin{aligned}
\bar{\delta}(\mathbf{h}) & =\frac{\sum_{j=1}^{M}(h(j)-j)^{+}}{\sum_{j=1}^{M}(M-j)}=\frac{2}{M(M-1)} \sum_{j=1}^{M}(h(j)-j)^{+} \\
& =\frac{2 M}{M-1} \sum_{j=1}^{M}\left(\frac{h(j)}{M}-\frac{j}{M}\right)^{+} \frac{1}{M} \\
\bar{\delta}[\mathbf{z}] & =\frac{2 M}{M-1} \int_{0}^{1}(\mathrm{z}(t)-t)^{+} \mathrm{d} t
\end{aligned}
$$

Theorem 6 below specializes Theorem 5 to the case of constant packet inter-arrival times, yielding approximate but accurate closed form rate delay tradeoffs for large $M$.

Theorem 6 (Approximate Rate Delay Tradeoff for Constant Inter Packet Arrival Times): The approximate (asymptotically accurate) rate delay tradeoff solving (20) for a normalized delay bound $\bar{d} \in[0,1]$ is given by

$$
\bar{\rho}_{\bar{d}}^{*}=\left(1-\log \left(\sqrt{\frac{M-1}{M} \bar{d}}\right)\right)^{-1} .
$$

Alternatively, the rate delay tradeoff for a normalized rate requirement $\bar{\rho} \in[0,1]$ is given by

$$
\bar{d}_{\bar{\rho}}^{*}=\frac{M}{M-1} \exp \left(2-\frac{2}{\bar{\rho}}\right) .
$$

The optimal decoding deadline function $z^{*}$ takes the form

$$
\mathbf{z}^{*}(t)= \begin{cases}\zeta & 0 \leq t \leq \zeta^{*} \\ t & \zeta^{*}<t \leq 1\end{cases}
$$

with $\zeta^{*}=\sqrt{\frac{M-1}{M} \bar{d}}$.

The proof is found in Appendix F. The approximate and exact rate delay tradeoffs are plotted together in Figure 5.
E. Trading Rate for Average Sum Delay with I.I.D. Arrival Times

The second special case we consider is that of i.i.d. packet arrival times $\left(t_{1}, \ldots, t_{M}\right)$ with density $\mathrm{f}$, cumulative distribution $F$, and inverse function to the cumulative distribution $\mathrm{F}^{-1}$. The asymptotic theory of order statistics ([47] Theorem 10.3 pp. 288) asserts that $\sqrt{M}\left(\tau_{M z}-\mathrm{F}^{-1}(z)\right)$ has an asymptotic normal distribution as $M \rightarrow \infty$. That is, provided $0<\mathrm{f}\left(\mathrm{F}^{-1}(z)\right)<\infty$ (an easily satisfied condition), then

$$
\sqrt{M}\left(\tau_{M z}-\mathrm{F}^{-1}(z)\right) \stackrel{M \rightarrow \infty}{\longrightarrow} \mathcal{N}\left(0, \frac{z(1-z)}{\left(\mathrm{f}\left(\mathrm{F}^{-1}(z)\right)\right)^{2}}\right) .
$$

It follows that for large $M, \mathbb{E}\left[\tau_{M \mathrm{z}(t)}\right] \approx \mathrm{F}^{-1}(\mathrm{z}(t))$ for each $t \in(0,1)$, and that the random variable $\tau_{M z(t)}$ is increasingly concentrated around its mean, and therefore $\mathbb{E}\left[\left(\tau_{M \mathrm{z}(t)}-t N T_{s}\right)^{+}\right] \approx\left(\mathrm{F}^{-1}(\mathrm{z}(t))-t N T_{s}\right)^{+}$. This implies that the large $M$ approximate form of the delay functional for the mean sum delay metric, i.e., $p=1$ in (19), is

$$
\bar{\delta}[\mathrm{z}] \approx \frac{\int_{0}^{1}\left(\mathrm{~F}^{-1}(\mathrm{z}(t))-t N T_{s}\right)^{+} \mathrm{d} t}{\int_{0}^{1} \mathbb{E}\left[\left(\tau_{M}-t N T_{s}\right)^{+}\right] \mathrm{d} t} .
$$

Here, the denominator may also be approximated through the asymptotic distribution of the extreme values ([47], Ch. 11), which after scaling can take only one of three forms.

To get a closed form expression for the rate delay tradeoff, we now specialize to the case where the arrival times $\left(t_{1}, \ldots, t_{M}\right)$ are i.i.d. exponential random variables with parameter $\lambda$. Symmetry suggests that the permutation distribution will be uniform, thereby selecting the capacity region solved in Theorem 2. We first develop an expression for the expected sum delay for fixed decoding deadlines $\mathbf{h}$, i.e., the expected value of (14) for $p=1$ and i.i.d. exponentials. We then develop an approximation for the normalized expected sum delay expressed (19) in terms of the functional $z$ appropriate for large $M$.

For finite $M$ the ordered packet arrival times $\left(\tau_{1}, \ldots, \tau_{M}\right)$ are order statistics, having marginal distributions

$$
\mathrm{f}\left(\tau_{j}\right)=j \lambda\left(\begin{array}{c}
M \\
j
\end{array}\right) \sum_{k=0}^{j-1}\left(\begin{array}{c}
j-1 \\
k
\end{array}\right)(-1)^{j-k-1} \mathrm{e}^{-(M-k) \lambda \tau_{j}},
$$

for $\tau_{j} \geq 0$ and $j \in\{1, \ldots, M\}$. Under frame decoding deadline constraints $\mathbf{h}$, the expected decoding delay of frame $i$ is then given by applying the definition of expectation with respect to this distribution, making a change of variables, and integrating by parts to get

$$
\begin{aligned}
& \mathbb{E}\left[\left(\tau_{h(i)}-i T_{s}\right)^{+}\right]= h(i)\left(\begin{array}{c}
M \\
h(i)
\end{array}\right) \sum_{k=0}^{h(i)-1}\left(\begin{array}{c}
h(i)-1 \\
k
\end{array}\right) \\
& \frac{(-1)^{h(i)-k-1} \mathrm{e}^{-(M-k) \lambda i T_{s}}}{\lambda(M-k)^{2}}
\end{aligned}
$$

for $i \in\{1, \ldots, N\}$. Summing these expected decoding delays 


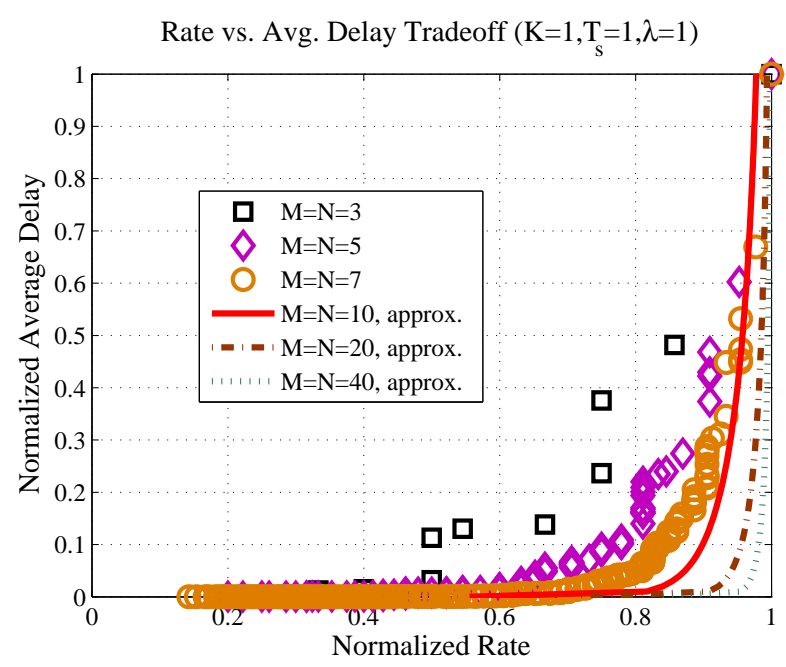

Fig. 6. Exact normalized rate delay tradeoffs for some small $M, N$ for the i.i.d. exponential arrival time case for $M=N=3,5,7$, and the approximated rate delay tradeoff via the calculus of variations for $M=N=$ $10,20,40$.

over each frame yields an expected overall delay under $\mathbf{h}$ of

$$
\begin{aligned}
\mathbb{E}\left[D_{1}(\mathbf{h})\right]= & \sum_{i=1}^{N} h(i)\left(\begin{array}{c}
M \\
h(i)
\end{array}\right) \sum_{k=0}^{h(i)-1}\left(\begin{array}{c}
h(i)-1 \\
k
\end{array}\right)(-1)^{h(i)-k-1} \\
& \frac{\mathrm{e}^{-(M-k) \lambda i T_{s}}}{\lambda(M-k)^{2}} .
\end{aligned}
$$

The optimal rate-delay tradeoff for small or moderate $M, N$ can be determined by search over all decoding deadline sequences $\mathbf{h}$ as in Theorem 4. Figure 6 plots some sample normalized rate delay tradeoffs.

For the exponential CDF $\mathrm{F}(x)=1-\mathrm{e}^{-\lambda x}$, the inverse CDF is $\mathrm{F}^{-1}(z)=-\lambda^{-1} \log (1-z)$. Substituting this inverse $\mathrm{CDF}$ into (26) yields

$$
\bar{\delta}[\mathbf{z}] \approx \frac{\int_{0}^{1}\left(-\lambda^{-1} \log (1-\mathrm{z}(t))-t N T_{s}\right)^{+} \mathrm{d} t}{\int_{0}^{1} \mathbb{E}\left[\left(\tau_{M}-t N T_{s}\right)^{+}\right] \mathrm{d} t}
$$

which may be used to obtain the following rate delay tradeoff.

Theorem 7 (Rate Delay Tradeoff for i.i.d. Exponential Packet Arrival Times): The approximate (asymptotically accurate) rate delay tradeoff solving (20) for a normalized delay bound $\bar{d} \in[0,1]$ is given by

$$
\bar{\rho}_{\bar{d}}^{*} \approx \lambda N T_{s}\left(\frac{(\gamma+\log M) \sqrt{\bar{d}}}{1-\mathrm{e}^{-\gamma \sqrt{d}} M^{-\sqrt{d}}}+\log \frac{\mathrm{e}^{\lambda N T_{s}}-1}{\mathrm{e}^{\gamma \sqrt{d}} M^{\sqrt{d}}-1}\right)^{-1}
$$

when $N>\frac{\gamma+\log M}{\lambda T_{s}}$ and

$$
\begin{aligned}
\bar{\rho}_{\bar{d}}^{*} \approx & \lambda N T_{s}\left[\frac{\sqrt{\left(2 \gamma+2 \log M-\lambda N T_{s}\right) \lambda N T_{s} \bar{d}}}{1-\exp \left(-\sqrt{\left(2 \gamma+2 \log M-\lambda N T_{s}\right) \lambda N T_{s} \bar{d}}\right)}\right. \\
& \left.+\log \frac{\exp \left(\lambda N T_{s}\right)-1}{\exp \left(\sqrt{\left(2 \gamma+2 \log M-\lambda N T_{s}\right) \lambda N T_{s} \bar{d}}\right)-1}\right]^{-1}
\end{aligned}
$$

when $N \leq \frac{\gamma+\log M}{\lambda T_{s}}$. Here in both cases $\gamma \approx 0.5772156649$ is the Euler Mascheroni constant. The associated optimal decoding deadline function takes the form

$$
\mathrm{z}^{*}(t)=\left\{\begin{array}{ll}
1-\mathrm{e}^{-\lambda N T_{s} \zeta^{*}}, & 0 \leq t \leq \zeta^{*} \\
1-\mathrm{e}^{-\lambda N T_{s} t}, & \zeta^{*}<t \leq 1
\end{array},\right.
$$

with

$$
\zeta^{*}=\left\{\begin{array}{cc}
\frac{(\gamma+\log M) \sqrt{d}}{\lambda N T_{s}} & N<\frac{\gamma+\log M}{\lambda T_{s}} \\
\sqrt{\left(\frac{2(\gamma+\log M)}{\lambda N T_{s}}-1\right) \bar{d}} N \geq \frac{\gamma+\log M}{\lambda T_{s}}
\end{array} .\right.
$$

The proof is found in Appendix G. Figure 6 shows some example approximate rate delay tradeoffs by plotting these expressions. The figure illustrates that significant reductions in normalized delay are achievable with only small or moderate reductions in normalized rate.

\section{Practical Delay Mitigating Codes}

This paper has addressed rate delay tradeoffs for multipath routed channels and random linear network coded channels. For multipath routed channels we have focused on the case where the packet reordering permutation is uniform over the set of all permutations. For random linear network coded channels we have focused on the case where the encoding matrices are selected uniformly over all matrices of a given rank. The relevant abstracted degraded broadcast channel capacity region for both cases is presented in §III, where we show the capacity regions of the two channels to be the same. This region is characterized by one linear constraint, and thus all capacity achieving strategies may be formed by time sharing (across "users" of the degraded broadcast channel) codes which transmit to only one of the users at maximal rate for that user. In particular, we may time share codes with rate vectors $\mathbf{r}_{j}=j K \mathbf{e}_{j}$ bits per channel use, with $\mathbf{e}_{j}$ the $j$ th column of the identity matrix to get any rate vector $\mathbf{r}$ in the capacity region of the uniform permutation channel or the uniform network coded channel. Capacity achieving codes with rate vectors $\mathbf{r}_{j}=j K \mathbf{e}_{j}$ exist for finite block lengths and appropriate $K$, and have the property in the multipath routed case that they encode $j$ packets of $K$ source bits to form at total of $M K$ encoded bits, from any subset of $j K$-bit packets of which the original source bits can be determined. Such codes and are called maximum distance separable (MDS) erasure codes. Alternatively, in the uniform network coded case, codes with rate vectors $\mathbf{r}_{j}=j K \mathbf{e}_{j}$ bits per channel use encode $j K$ source bits into $M$ packets of $K$ encoded bits, is such a way that the source bits can be determined from any rank $j$ linear combination of the $M$ packets. Such codes are called rank metric maximum distance separable codes [19]. The act of time sharing such maximum distance separable codes is commonly referred to as priority encoded transmission (PET) after [11], [36], and their rank metric counterparts have recently been investigated [12], [19]. Thus, in the uniform case studied in detail in this article, codes exist which achieve the introduced rate delay tradeoff, and they are PET codes in the permutation channel case, and rank metric PET codes in the network coded case. In the PET construction, it is left up to the user what rates the component 
MDS codes should have, so that the PET name is given to any construction which time shares MDS codes, regardless of the component rates. This article provides the necessary analysis for an end user who needs a particular bounded delay to determine the component code rates of a time shared collection of MDS codes in order to satisfy this delay. Because the PET construction in [11] is slightly different from the one presented here, namely in that it allows the number of packets $M$ to be dictated by other problem variables, while $M$ is fixed here, and we allow the number of bits per packet $K$ to be dictated by other problem variables, we will provide a brief discussion of how to construct practical PET codes in this section.

In particular, let's discuss how to obtain a code achieving a point on the rate delay tradeoff. Suppose we have selected a delay bound $d$, and the rate vector $\mathbf{r}_{d}^{*}$ bits per channel use for the degraded broadcast channel attains the maximal bit rate for this delay, as in (1) and (2). First, we encode the (temporally) first $R_{1}(d)$ bits of the source data into $R_{1}(d) M$ bits with a MDS code. These bits are then divided up into $M$ equally sized contiguous consecutive blocks of length $R_{1}(d)$, and one such block is placed at the beginning of each of the $M$ packets. Second, we encode the next $R_{2}(d)$ bits of the source data into $\frac{R_{2}(d) M}{2}$ encoded bits using a MDS code. These bits are then divided up equally (and contiguously and consecutively) into blocks of size $\frac{R_{2}}{2}$ bits and one such block is placed in each of the $M$ packets. The construction continues, i.e., at the $j$ th step the next $R_{j}(d)$ bits of source data are encoded into $\frac{R_{j}(d) M}{j}$ encoded bits using a MDS code, and these are broken up into $M$ contiguous blocks of $\frac{R_{j}(d)}{j}$ bits, with one such block placed in one of each of the $M$ packets.

The discussion above glosses over the quantization of rates that must occur and the selection of an appropriate packet length $K$. If we are using Reed Solomon codes over $\operatorname{GF}(q)$ with $q=2^{m}$ as the MDS codes, for instance, then the number of source bits $R_{j}(d)$ must equal $\left(2^{m}-1-2 t\right) m$ for some integer $t_{j}$, and the number of encoded bits $\frac{R_{j}(d) M}{j}$ must equal $\left(2^{m}-1\right) m$ [48]. Furthermore, $\frac{\left(2^{m}-1\right) m}{M}$ must be an integer multiple of $m$, so that the blocks of information to go into the different packets break up at boundaries between different elements of the finite field. One can always select the packet length $K, t_{j}$, and the field size $2^{m}$ to obey these conditions and arbitrarily closely approximate the rate delay tradeoff. In particular, due to the form of the rate regions of the degraded broadcast channels (6)-(8), choosing a different $K^{\prime}$ for the same delay constraint simply scales the optimal rate vector associated with this delay to $\mathbf{r}^{\prime}(d)=\frac{K^{\prime}}{K} \mathbf{r}(d)$, and thus the total rate $\rho^{\prime}(d)=\frac{K^{\prime}}{K} \rho(d)$. Thus, first select any point on the normalized rate delay tradeoff curve, e.g. by taking the associated $K$ to be $K=1$. Take the rate vector $\mathbf{r}$ associated with this (or a sufficiently close approximation to it that has all elements as rational numbers if some of its elements are not rational numbers), and scale it to $\mathbf{r} K^{\prime}$, with $K^{\prime}$ a natural number selected so that the $j$ element of the vector $\mathbf{r} K^{\prime}$ is a natural number divisible by $j m$. For instance, given a rational $\mathbf{r}$ it suffices (but is not necessary) to take $K^{\prime}=G m M$ where $G$ is the least common multiple of the denominators of the rational numbers to achieve this. Then, for the $j$ th component code with specified rate $R_{j}(d)$, select $t_{j}$ to be

$$
t_{j}=\left\lceil\frac{\left(2^{m}-1\right)\left(1-\frac{j}{M}\right)}{2}\right\rceil
$$

This will give a normalized rate and delay arbitrarily close to the one originally selected as $m$ gets large, as well as an associated rate vector that is in the achievable rate region for any $m$.

Finally, it is important to note that, for some other, nonuniform cases, the rate regions of the degraded broadcast channels introduced in this paper are different, and PET codes do not achieve the rate delay tradeoffs, because they are not capacity achieving strategies for these degraded broadcast channels. The rate delay tradeoff framework in this paper, however, still provides an upper bound on the achievable bit rate given a delay in these cases.

\section{INCORPORATING FEEDBACK AND PACKET LOSS}

This paper has employed forward erasure correction based delay mitigation. However, many modern communications networks are bidirectional. One could then ask if better rate delay tradeoffs could be achieved by the use of ARQ-like schemes incorporating feedback. Surprisingly, the rate delay tradeoffs presented are the same, even if feedback is used. Fundamentally, this is because feedback does not change the capacity region of the discrete memoryless degraded broadcast channel [49]. Applying this fact to our case, feedback could only affect the rate delay tradeoffs through the associated abstracted degraded broadcast channel's capacity region. Thus, while the codes we have discussed have been forward erasure correction based, a scheme incorporating feedback is subject to the same rate delay tradeoffs.

An additional natural extension points out the fact that real networks also exhibit (non-negligible) packet loss in addition to packet reorderings and delays. Packet losses could be incorporated in the present model by simply having any lost packets arrive at a very large time $\tau \approx \infty$. A small delay bound would generate rate delay tradeoff that would simply not require all packets to be received before all of the source frames can be decoded. Indeed, even without incorporating such large delays into the model directly, they happen in the i.i.d. $\left\{t_{j}\right\}$ case in the limit as $M \rightarrow \infty$ (which is the limit we investigated) due to the growth of the maximal order statistic. The optimal decoding deadlines found via the calculus of variations continous approximation in this case already do not require all of the encoded packets to arrive before all of the source frames may be decoded (effectively already handling packet loss).

An alternative approach at including packet loss by incorporating random numbers of packet losses would allow for an opportunity to discuss a more classic information theoretic way of studying rate delay tradeoffs: through error exponents. Note that for the abstracted degraded broadcast channels presented in this paper, the error exponents are infinite (more precisely: after a critical block length of one channel use it is possible to create capacity achieving codes that have zero probability of error), and thus a classic study of rate delay 
tradeoffs through the use of error exponents would not have made sense. However, as we have seen, even with infinite error exponents for the associated abstracted degraded broadcast channel, there is still a rigorous and important notion of a rate delay tradeoff. If random numbers of packet losses were incorporated, the error exponents would no longer be infinite, and a rate delay tradeoff analysis through error exponents should become feasible. It would be interesting to see how the present results could be melded into such a setup, and is part of our plans for future work.

\section{CONCLUSIONS}

This paper has addressed the optimal rate delay tradeoff in multipath routed and network coded networks. In both cases we have focused on the uniform distribution case: uniformly selected packet reordering for multipath routed networks, and uniformly selected encoding matrices for network coded networks. Both cases are modeled as an abstracted degraded broadcast channel, their capacity regions are shown to be the same, and the region is specified by a single linear inequality. The rate delay tradeoff is formulated as a nonlinear continuous optimization problem, but we demonstrate the problem is in fact equivalent to an integer optimization problem, suitable for solution via enumeration for moderate $M, N$. For large $M, N$ we propose an approximate solution using the calculus of variations. We solve this variational problem and obtain explicit expressions for rate delay tradeoffs for the two special cases of constant packet inter-arrival times and i.i.d. exponentially distributed arrival times. We propose the timeshared use of practical finite blocklength capacity achieving codes: PET codes for multipath routed networks, and rank-metric MDS component codes for linear network coded networks. The rates of these codes are dictated by the fundamental rate delay tradeoffs we present, and are determined by the specified delay bound.

\section{APPENDIX A}

\section{MATHEMATICAL NOTATION}

See Table I.

\section{APPENDIX B}

PROOF OF PROP. 1

We begin with the general expression for receiver $k$

$$
\begin{aligned}
& \mathrm{I}\left(\mathbf{y}_{k} ; \mathbf{u}_{k} \mid \mathbf{u}_{k-1}\right) \\
= & \mathrm{I}\left(\left(\pi(1), \mathbf{x}_{\pi(1)}, \ldots, \pi(k), \mathbf{x}_{\pi(k)}\right) ; \mathbf{u}_{k} \mid \mathbf{u}_{k-1}\right) \\
= & \mathrm{H}\left(\pi(1), \mathbf{x}_{\pi(1)}, \ldots, \pi(k), \mathbf{x}_{\pi(k)} \mid \mathbf{u}_{k-1}\right) \\
& -\mathrm{H}\left(\pi(1), \mathbf{x}_{\pi(1)}, \ldots, \pi(k), \mathbf{x}_{\pi(k)} \mid \mathbf{u}_{k}\right) \\
= & \mathbf{H}\left(\pi(1), \ldots, \pi(k) \mid \mathbf{u}_{k-1}\right)-\mathrm{H}\left(\pi(1), \ldots, \pi(k) \mid \mathbf{u}_{k}\right) \\
& +\mathbf{H}\left(\mathbf{x}_{\pi(1)}, \ldots, \mathbf{x}_{\pi(k)} \mid \pi(1), \ldots, \pi(k), \mathbf{u}_{k-1}\right) \\
& -\mathbf{H}\left(\mathbf{x}_{\pi(1)}, \ldots, \mathbf{x}_{\pi(k)} \mid \pi(1), \ldots, \pi(k), \mathbf{u}_{k}\right)
\end{aligned}
$$

$$
\begin{aligned}
= & \mathrm{H}(\pi(1), \ldots, \pi(k))-\mathrm{H}(\pi(1), \ldots, \pi(k)) \\
& +\mathrm{H}\left(\mathbf{x}_{\pi(1)}, \ldots, \mathbf{x}_{\pi(k)} \mid \pi(1), \ldots, \pi(k), \mathbf{u}_{k-1}\right) \\
& -\mathrm{H}\left(\mathbf{x}_{\pi(1)}, \ldots, \mathbf{x}_{\pi(k)} \mid \pi(1), \ldots, \pi(k), \mathbf{u}_{k}\right) \\
= & \sum_{\tilde{\pi} \in \Pi} \mathrm{p}(\tilde{\pi})\left(\mathrm{H}\left(\mathbf{x}_{\tilde{\pi}(1)}, \ldots, \mathbf{x}_{\tilde{\pi}(k)} \mid \mathbf{u}_{k-1}\right)\right. \\
- & \left.\mathrm{H}\left(\mathbf{x}_{\tilde{\pi}(1)}, \ldots, \mathbf{x}_{\tilde{\pi}(k)} \mid \mathbf{u}_{k}\right)\right)
\end{aligned}
$$

Here, (28) substitutes in the definition of $\mathbf{y}_{k}$ and (29) is the definition of condition mutual information together with the fact that $\mathbf{u}_{1} \rightarrow \mathbf{u}_{2} \rightarrow \mathbf{u}_{M-1} \rightarrow \mathbf{x}$ form a Markov chain. Next, (30) expresses a joint entropy as a marginal entropy plus a conditional entropy (e.g., $\mathrm{H}(U, V)=\mathrm{H}(U)+$ $\mathrm{H}(V \mid U))$. The process selecting the random reordering variables $(\pi(1), \ldots, \pi(M))$, which comes from the discrete memoryless channel, has nothing to do with the contents of the input block of packets $\mathrm{x}$ or the message random variables, so these are independent of one another, giving (31). Substituting in the definition of conditional entropy and recognizing that the contents of the received packets only depend on the arrival order through their index then gives the desired equation (32). We emphasize the distinction in the last step between the random variables $\pi$ and a particular realization (permutation) $\tilde{\pi} \in \Pi$, for $\Pi$ the set of permutations of the numbers $1, \ldots, M$. The equivalences for the other two equations (6) and (8) follow from essentially the same chain of arguments.

\section{APPENDIX C \\ Proof of THEOREM 2: UNIFORM PERMUTATION CHANNEL CAPACITY REGION}

Han's inequality ([50] Theorem 17.6 .1 pp. 668, citing [51]) states:

$$
\frac{1}{k\left(\begin{array}{c}
M \\
k
\end{array}\right)} \sum_{S:|S|=k} \mathrm{H}\left(X_{S}\right) \leq \frac{1}{(k-1)\left(\begin{array}{c}
M \\
k-1
\end{array}\right)} \sum_{S:|S|=k-1} \mathrm{H}\left(X_{S}\right)
$$

for random variables $X_{1}, \ldots, X_{M}$ and for each $k=2, \ldots, M$ and where $X_{S}$ is shorthand for $X_{i}, i \in S$. Prop. 3 specializes Han's inequality to the uniform permutation channel.

\section{Proposition 3: For the uniform permutation channel}

$$
\begin{gathered}
\frac{1}{k} \sum_{\tilde{\pi} \in \Pi} \mathrm{p}(\tilde{\pi}) \mathrm{H}\left(\mathbf{x}_{\tilde{\pi}(1)}, \ldots, \mathbf{x}_{\tilde{\pi}(k)} \mid \mathbf{u}_{k-1}\right) \leq \\
\frac{1}{k-1} \sum_{\tilde{\pi} \in \Pi} \mathrm{p}(\tilde{\pi}) \mathrm{H}\left(\mathbf{x}_{\tilde{\pi}(1)}, \ldots, \mathbf{x}_{\tilde{\pi}(k-1)} \mid \mathbf{u}_{k-1}\right),
\end{gathered}
$$

for each $k \in\{2, \ldots, M\}$.

Consider the left side of (34). By assumption the distribution on the permutations is uniform: $\mathrm{p}(\tilde{\pi})=\frac{1}{|\Pi|}$ for each $\tilde{\pi} \in \Pi$. There are $M$ ! different permutations, and hence $\mathrm{p}(\tilde{\pi})=\frac{1}{M !}$. However, the entropy depends only upon the unordered set of the first $k$ elements of each permutation, $\{\tilde{\pi}(1), \ldots, \tilde{\pi}(k)\}$. Define $\mathcal{C}_{k}$ to be the set of all subsets from $\{1, \ldots, M\}$ of size $k$. We may then group together all $\tilde{\pi}$ with a common unordered set of first $k$ elements. There are $\left(\begin{array}{c}M \\ k\end{array}\right)$ distinct such sets, and 
TABLE I

MATHEMATICAL NOTATION

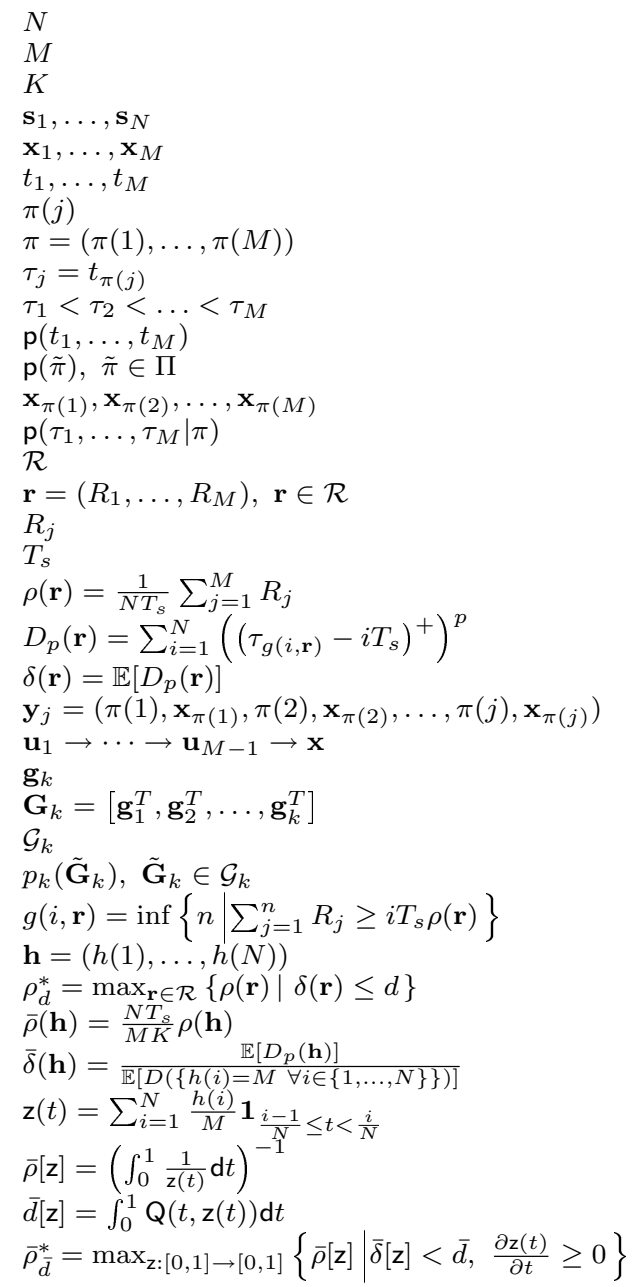

number of source frames

number of packets sent over network

length in bits of each packet

temporally ordered frames from a multimedia source encoder

packets sent over network

packet arrival times

index of the transmitted packet that is the $j$ th received packet

permutation of the packets capturing packet reordering

time of $j$ th received packet

packet arrival times in order of reception

joint distribution on packet arrival times

distribution on the packet reordering permutation

packets in order of reception

joint distribution on receiver-ordered packet arrival times

capacity region of degraded broadcast channel

a rate vector in the capacity region

amount of new information (in bits) available upon the $j$ th received packet desired time between successive playback of the temporally ordered frames overall rate at which data is decoded at the source decoder

overall random delay for rate vector $\mathbf{r}$

overall expected delay associated with rate vector $\mathbf{r}$

cumulative information available at destination upon the $j$ th received packet dummy discrete random variables characterizing the DBC capacity region $\mathcal{R}$ random global encoding vector (using network coding) of the $k$ th received packet random global encoding matrix

set of global encoding matrices of rank $k$ from $\operatorname{GF}(q)^{k \times M}$

probability distribution on the set of global encoding matrices

\# of packets that must be received before the first $i$ frames may be decoded

decoding deadline for each $g(i, \mathbf{r})$; i.e., each $g(i, \mathbf{r}) \leq h(i)$

max. feasible overall rate subject to a bound $d$ on expected overall delay

overall rate $\rho$ normalized to lie in $[0,1]$ for decoding deadlines $\mathbf{h}$

overall delay normalized to lie in $[0,1]$ for decoding deadlines $\mathbf{h}$

zero order hold piecewise continuous fen. $\mathbf{z}:[0,1] \rightarrow[0,1]$ s.t. $h(i)=M z(i / N)$

overall rate $\rho$ normalized to lie in $[0,1]$ for approximate decoding deadlines $z$ overall norm. delay for approx. decoding deadlines $\mathbf{z}(\cdot)$ with integrand $\mathbf{Q}(t, \mathbf{z}(t))$

optimal overall normalized rate subject to overall normalized delay requirement $\bar{d}$.

thus each group of terms has $M ! /\left(\begin{array}{c}M \\ k\end{array}\right)$ elements. This allows

$$
\begin{aligned}
& \frac{1}{k} \sum_{\tilde{\pi} \in \Pi} \mathrm{p}(\tilde{\pi}) \mathrm{H}\left(\mathbf{x}_{\tilde{\pi}(1)}, \ldots, \mathbf{x}_{\tilde{\pi}(k)} \mid \mathbf{u}_{k-1}\right) \\
= & \frac{1}{k\left(\begin{array}{c}
M \\
k
\end{array}\right)} \sum_{\{\tilde{\pi}(1), \ldots, \tilde{\pi}(k)\} \in \mathcal{C}_{k}} \mathrm{H}\left(\mathbf{x}_{\tilde{\pi}(1)}, \ldots, \mathbf{x}_{\tilde{\pi}(k)} \mid \mathbf{u}_{k-1}\right) .
\end{aligned}
$$

Repeating this procedure for $k-1$ (with the entropy still conditioned on $\mathbf{u}_{k-1}$, as above) gives

$$
\begin{gathered}
\frac{1}{k-1} \sum_{\tilde{\pi} \in \Pi} \mathrm{p}(\tilde{\pi}) \mathrm{H}\left(\mathbf{x}_{\tilde{\pi}(1)}, \ldots, \mathbf{x}_{\tilde{\pi}(k-1)} \mid \mathbf{u}_{k-1}\right)= \\
\sum_{\{\tilde{\pi}(1), \ldots, \tilde{\pi}(k-1)\} \in \mathcal{C}_{k-1}} \frac{\mathrm{H}\left(\mathbf{x}_{\tilde{\pi}(1)}, \ldots, \mathbf{x}_{\tilde{\pi}(k-1)} \mid \mathbf{u}_{k-1}\right)}{(k-1)\left(\begin{array}{c}
M \\
k-1
\end{array}\right)} .
\end{gathered}
$$

The proposition follows by Han's inequality (33).

Proposition 4: For the uniform permutation channel:

$$
\begin{aligned}
\sum_{j=1}^{k} \frac{R_{j}}{j} \leq & \sum_{\tilde{\pi} \in \Pi} \mathrm{p}(\tilde{\pi}) \mathrm{H}\left(\mathbf{x}_{\tilde{\pi}(1)}\right) \\
& -\frac{1}{k} \sum_{\tilde{\pi} \in \Pi} \mathrm{p}(\tilde{\pi}) \mathrm{H}\left(\mathbf{x}_{\tilde{\pi}(1)}, \ldots, \mathbf{x}_{\tilde{\pi}(k)} \mid \mathbf{u}_{k}\right),
\end{aligned}
$$

for $k \in\{1, \ldots, M\}$.

The proof is by induction on $k$. The base case $k=1$ is true by (6) in Prop. 1:

$$
R_{1} \leq \sum_{\tilde{\pi} \in \Pi} \mathrm{p}(\tilde{\pi}) \mathrm{H}\left(\mathbf{x}_{\tilde{\pi}(1)}\right)-\sum_{\tilde{\pi} \in \Pi} \mathrm{p}(\tilde{\pi}) \mathrm{H}\left(\mathbf{x}_{\tilde{\pi}(1)} \mid \mathbf{u}_{1}\right)
$$

Suppose the proposition is true for $k-1$ :

$$
\begin{aligned}
\sum_{j=1}^{k-1} \frac{R_{j}}{j} \leq & \sum_{\tilde{\pi} \in \Pi} \mathrm{p}(\tilde{\pi}) \mathrm{H}\left(\mathbf{x}_{\tilde{\pi}(1)}\right) \\
& -\frac{1}{k-1} \sum_{\tilde{\pi} \in \Pi} \mathrm{p}(\tilde{\pi}) \mathrm{H}\left(\mathbf{x}_{\tilde{\pi}(1)}, \ldots, \mathbf{x}_{\tilde{\pi}(k-1)} \mid \mathbf{u}_{k-1}\right) .
\end{aligned}
$$

The proposition follows by successive application of the 
induction hypothesis, (7) in Prop. 1, and Prop. 3:

$$
\begin{aligned}
& \sum_{j=1}^{k} \frac{R_{j}}{j}=\frac{R_{k}}{k}+\sum_{j=1}^{k-1} \frac{R_{j}}{j} \leq \frac{R_{k}}{k}+\sum_{\tilde{\pi} \in \Pi} \mathrm{p}(\tilde{\pi}) \mathrm{H}\left(\mathbf{x}_{\tilde{\pi}(1)}\right) \\
& -\frac{1}{k-1} \sum_{\tilde{\pi} \in \Pi} \mathrm{p}(\tilde{\pi}) \mathrm{H}\left(\mathbf{x}_{\tilde{\pi}(1)}, \ldots, \mathbf{x}_{\tilde{\pi}(k-1)} \mid \mathbf{u}_{k-1}\right) \\
\leq & \frac{1}{k} \sum_{\tilde{\pi} \in \Pi} \mathrm{p}(\tilde{\pi})\left(\mathrm{H}\left(\mathbf{x}_{\tilde{\pi}(1)}, \ldots, \mathbf{x}_{\tilde{\pi}(k)} \mid \mathbf{u}_{k-1}\right)\right. \\
& \left.-\mathrm{H}\left(\mathbf{x}_{\tilde{\pi}(1)}, \ldots, \mathbf{x}_{\tilde{\pi}(k)} \mid \mathbf{u}_{k}\right)\right)+\sum_{\tilde{\pi} \in \Pi} \mathrm{p}(\tilde{\pi}) \mathrm{H}\left(\mathbf{x}_{\tilde{\pi}(1)}\right) \\
& -\frac{1}{k-1} \sum_{\tilde{\pi} \in \Pi} \mathrm{p}(\tilde{\pi}) \mathrm{H}\left(\mathbf{x}_{\tilde{\pi}(1)}, \ldots, \mathbf{x}_{\tilde{\pi}(k-1)} \mid \mathbf{u}_{k-1}\right) \\
\leq & \frac{1}{k-1} \sum_{\tilde{\pi} \in \Pi} \mathrm{p}(\tilde{\pi}) \mathrm{H}\left(\mathbf{x}_{\tilde{\pi}(1)}, \ldots, \mathbf{x}_{\tilde{\pi}(k-1)} \mid \mathbf{u}_{k-1}\right) \\
& -\frac{1}{k} \sum_{\tilde{\pi} \in \Pi} \mathrm{p}(\tilde{\pi}) \mathrm{H}\left(\mathbf{x}_{\tilde{\pi}(1)}, \ldots, \mathbf{x}_{\tilde{\pi}(k)} \mid \mathbf{u}_{k}\right)+\sum_{\tilde{\pi} \in \Pi} \mathrm{p}(\tilde{\pi}) \mathrm{H}\left(\mathbf{x}_{\tilde{\pi}(1)}\right) \\
& -\frac{1}{k-1} \sum_{\tilde{\pi} \in \Pi} \mathrm{p}(\tilde{\pi}) \mathrm{H}\left(\mathbf{x}_{\tilde{\pi}(1)}, \ldots, \mathbf{x}_{\tilde{\pi}(k-1)} \mid \mathbf{u}_{k-1}\right) \\
= & \sum_{\tilde{\pi} \in \Pi} \mathrm{p}(\tilde{\pi}) \mathrm{H}\left(\mathbf{x}_{\tilde{\pi}(1)}\right)-\frac{1}{k} \sum_{\tilde{\pi} \in \Pi} \mathrm{p}(\tilde{\pi}) \mathrm{H}\left(\mathbf{x}_{\tilde{\pi}(1)}, \ldots, \mathbf{x}_{\tilde{\pi}(k)} \mid \mathbf{u}_{k}\right)
\end{aligned}
$$

This proves Prop. 4. The converse in Theorem 2 follows from Prop. 4 by setting $k=M$ and by noting that $\sum_{\tilde{\pi} \in \Pi} \mathrm{p}(\tilde{\pi}) \mathrm{H}\left(\mathbf{x}_{\tilde{\pi}(1)}\right) \leq K$ and that $\mathrm{H}\left(\mathbf{x}_{\tilde{\pi}(1)}, \ldots, \mathbf{x}_{\tilde{\pi}(M)} \mid \mathbf{u}_{M}\right)=0$. The remainder of the proof must show that these rates are achievable, i.e., that the inequality is tight. This may be established by noting that the capacity region is described by a single inequality. Furthermore, the corner points $\mathbf{r}_{j}=j K \mathbf{e}_{j}$ of this single inequality described rate region can be achieved using a capacity achieving codes for the point to point channel between the source and the $i$ th reciever alone. These codes may then be time shared to yield all points on the boundary of the capacity region. The code for the $j$ th receiver alone (which has rate point $\mathbf{r}_{j}=j K \mathbf{e}_{j}$ ) exists by the standard proof of the channel capacity for a discrete memoryless point to point channel, e.g., by employing random codes and jointly typical sequence decoding.

\section{APPENDIX D}

\section{Proof of Theorem 3: Uniform Network Coded Channel CAPACITY REgion}

The proof of the theorem depends upon the following proposition. Note that all matrices discussed below have elements in the finite field $\operatorname{GF}(q)$.

Proposition 5: For the uniform network coded degraded broadcast channel

$$
\begin{gathered}
\frac{1}{k} \sum_{\tilde{\mathbf{G}}_{k} \in \mathcal{G}_{k}} \mathrm{p}\left(\tilde{\mathbf{G}}_{k}\right) \mathbf{H}\left(\tilde{\mathbf{G}}_{k} \mathbf{x} \mid \mathbf{u}_{k-1}\right) \leq \\
\frac{1}{k-1} \sum_{\tilde{\mathbf{G}}_{k-1} \in \mathcal{G}_{k-1}} \mathrm{p}\left(\tilde{\mathbf{G}}_{k-1}\right) \mathbf{H}\left(\tilde{\mathbf{G}}_{k-1} \mathbf{x} \mid \mathbf{u}_{k-1}\right),
\end{gathered}
$$

for $k \in\{2, \ldots, M\}$.
Consider the left side of (38). We decompose $\mathrm{p}\left(\tilde{\mathbf{G}}_{k}\right)$ as:

$$
\mathrm{p}\left(\tilde{\mathbf{G}}_{k}\right)=\sum_{\tilde{\mathbf{G}}_{M} \in \mathcal{G}_{M}} \mathrm{p}\left(\tilde{\mathbf{G}}_{k} \mid \tilde{\mathbf{G}}_{M}\right) \mathrm{p}\left(\tilde{\mathbf{G}}_{M}\right) .
$$

By the uniform assumption $\mathrm{p}\left(\tilde{\mathbf{G}}_{M}\right)=\frac{1}{\left|\mathcal{G}_{M}\right|}$ for each $\tilde{\mathbf{G}}_{M} \in$ $\mathcal{G}_{M}$. Substitution and exchanging the order of summation yields:

$$
\begin{gathered}
\frac{1}{k} \sum_{\tilde{\mathbf{G}}_{k} \in \mathcal{G}_{k}} \mathrm{p}\left(\tilde{\mathbf{G}}_{k}\right) \mathrm{H}\left(\tilde{\mathbf{G}}_{k} \mathbf{x} \mid \mathbf{u}_{k-1}\right) \\
=\frac{1}{\left|\mathcal{G}_{M}\right|} \frac{1}{k} \sum_{\tilde{\mathbf{G}}_{k} \in \mathcal{G}_{k}}\left(\sum_{\tilde{\mathbf{G}}_{M} \in \mathcal{G}_{M}} \mathrm{p}\left(\tilde{\mathbf{G}}_{k} \mid \tilde{\mathbf{G}}_{M}\right)\right) \mathrm{H}\left(\tilde{\mathbf{G}}_{k} \mathbf{x} \mid \mathbf{u}_{k-1}\right) \\
=\frac{1}{\left|\mathcal{G}_{M}\right|} \sum_{\tilde{\mathbf{G}}_{M} \in \mathcal{G}_{M}} \frac{1}{k} \sum_{\tilde{\mathbf{G}}_{k} \in \mathcal{G}_{k}} \mathrm{p}\left(\tilde{\mathbf{G}}_{k} \mid \tilde{\mathbf{G}}_{M}\right) \mathrm{H}\left(\tilde{\mathbf{G}}_{k} \mathbf{x} \mid \mathbf{u}_{k-1}\right)
\end{gathered}
$$

The conditional distribution $\mathrm{p}\left(\tilde{\mathbf{G}}_{k} \mid \tilde{\mathbf{G}}_{M}\right)$ is the probability of randomly selecting (up to a row reordering) the $k \times M$ submatrix $\tilde{\mathbf{G}}_{k}$ from the $M \times M$ matrix $\tilde{\mathbf{G}}_{M}$ :

$$
\mathrm{p}\left(\tilde{\mathbf{G}}_{k} \mid \tilde{\mathbf{G}}_{M}\right)=\left\{\begin{array}{ll}
\frac{1}{\left(\begin{array}{c}
M \\
k
\end{array}\right)}, & \tilde{\mathbf{G}}_{k} \subseteq \tilde{\mathbf{G}}_{M} \\
0, & \text { else }
\end{array} .\right.
$$

This allows:

$$
\begin{array}{r}
\frac{1}{k} \sum_{\tilde{\mathbf{G}}_{k} \in \mathcal{G}_{k}} \mathrm{p}\left(\tilde{\mathbf{G}}_{k}\right) \mathbf{H}\left(\tilde{\mathbf{G}}_{k} \mathbf{x} \mid \mathbf{u}_{k-1}\right)=\frac{1}{\left|\mathcal{G}_{M}\right|} \sum_{\tilde{\mathbf{G}}_{M} \in \mathcal{G}_{M}} \frac{1}{k\left(\begin{array}{c}
M \\
k
\end{array}\right)} \\
\sum_{\tilde{\mathbf{G}}_{k} \in \mathcal{G}_{k}: \tilde{\mathbf{G}}_{k} \subseteq \tilde{\mathbf{G}}_{M}} \mathbf{H}\left(\tilde{\mathbf{G}}_{k} \mathbf{x} \mid \mathbf{u}_{k-1}\right)
\end{array}
$$

The inner sum over all $\tilde{\mathbf{G}}_{k} \in \mathcal{G}_{k}: \tilde{\mathbf{G}}_{k} \subseteq \tilde{\mathbf{G}}_{M}$ is the same as summing over all subsets of $\{1, \ldots, M\}$ of size $k$ and selecting the corresponding rows from $\tilde{\mathbf{G}}_{M}$. That is, define $\left[\tilde{\mathbf{G}}_{M}\right]_{\mathcal{S}}$ to be the rows from $\tilde{\mathbf{G}}_{M}$ with indices in $\mathcal{S}$, where $\mathcal{S} \subseteq\{1, \ldots, M\}$ and $|\mathcal{S}|=k$. This yields:

$$
\begin{array}{r}
\frac{1}{k} \sum_{\tilde{\mathbf{G}}_{k} \in \mathcal{G}_{k}} \mathrm{p}\left(\tilde{\mathbf{G}}_{k}\right) \mathbf{H}\left(\tilde{\mathbf{G}}_{k} \mathbf{x} \mid \mathbf{u}_{k-1}\right)=\frac{1}{\left|\mathcal{G}_{M}\right|} \sum_{\tilde{\mathbf{G}}_{M} \in \mathcal{G}_{M}} \frac{1}{k\left(\begin{array}{c}
M \\
k
\end{array}\right)} \\
\sum_{\mathcal{S} \subset\{1, \ldots, M\}:|\mathcal{S}|=k} \mathbf{H}\left(\left[\tilde{\mathbf{G}}_{M}\right]_{\mathcal{S}} \mathbf{x} \mid \mathbf{u}_{k-1}\right) .
\end{array}
$$

Finally, for each $\mathbf{x}$ and $\tilde{\mathbf{G}}_{M}$ define the $M$ random variables $\mathbf{q}=\tilde{\mathbf{G}}_{M} \mathbf{x}$ and the corresponding subset $\mathbf{q}_{\mathcal{S}}=\left[\tilde{\mathbf{G}}_{M} \mathbf{x}\right]_{\mathcal{S}}$ formed by selecting elements from $\mathbf{q}$ with indices in $\mathcal{S}$. This allows:

$$
\begin{array}{r}
\frac{1}{k} \sum_{\tilde{\mathbf{G}}_{k} \in \mathcal{G}_{k}} \mathrm{p}\left(\tilde{\mathbf{G}}_{k}\right) \mathrm{H}\left(\tilde{\mathbf{G}}_{k} \mathbf{x} \mid \mathbf{u}_{k-1}\right)=\frac{1}{\left|\mathcal{G}_{M}\right|} \sum_{\tilde{\mathbf{G}}_{M} \in \mathcal{G}_{M}} \frac{1}{k\left(\begin{array}{c}
M \\
k
\end{array}\right)} \\
\sum_{\mathcal{S} \subset\{1, \ldots, M\}:|\mathcal{S}|=k} \mathrm{H}\left(\mathbf{q}_{\mathcal{S}} \mid \mathbf{u}_{k-1}\right) .
\end{array}
$$

Performing the same sequence of steps for $k-1$ (still conditioning on $\mathbf{u}_{k-1}$, as above) gives:

$$
\begin{gathered}
\frac{1}{k-1} \sum_{\tilde{\mathbf{G}}_{k-1} \in \mathcal{G}_{k-1}} \mathbf{p}\left(\tilde{\mathbf{G}}_{k-1}\right) \mathrm{H}\left(\tilde{\mathbf{G}}_{k-1} \mathbf{x} \mid \mathbf{u}_{k-1}\right)= \\
\frac{1}{\left|\mathcal{G}_{M}\right|} \sum_{\tilde{\mathbf{G}}_{M} \in \mathcal{G}_{M}} \frac{1}{(k-1)\left(\begin{array}{c}
M \\
k-1
\end{array}\right)} \sum_{\mathcal{S} \subset\{1, \ldots, M\}:|\mathcal{S}|=k-1} \mathbf{H}\left(\mathbf{q}_{\mathcal{S}} \mid \mathbf{u}_{k-1}\right) .
\end{gathered}
$$


Applying Han's inequality (33) for each $\tilde{\mathbf{G}}_{M}$ completes the proof.

Proposition 6: For the uniform network coded degraded broadcast channel:

$\sum_{j=1}^{k} \frac{R_{j}}{j} \leq \sum_{\tilde{\mathbf{G}}_{1} \in \mathcal{G}_{1}} \mathrm{p}\left(\tilde{\mathbf{G}}_{1}\right) \mathrm{H}\left(\tilde{\mathbf{G}}_{1} \mathbf{x}\right)-\sum_{\tilde{\mathbf{G}}_{k} \in \mathcal{G}_{k}} \frac{\mathrm{p}\left(\tilde{\mathbf{G}}_{k}\right)}{k} \mathrm{H}\left(\tilde{\mathbf{G}}_{k} \mathbf{x} \mid \mathbf{u}_{k}\right)$,

for $k \in\{1, \ldots, M\}$.

The proof is by induction on $k$ and is identical to the proof of Prop. 4 (mutatis mutandis), but is included here for completeness. The base case $k=1$ is true by (10):

$$
R_{1} \leq \sum_{\tilde{\mathbf{G}}_{1} \in \mathcal{G}_{1}} \mathrm{p}\left(\tilde{\mathbf{G}}_{1}\right) \mathrm{H}\left(\tilde{\mathbf{G}}_{1} \mathbf{x}\right)-\sum_{\tilde{\mathbf{G}}_{1} \in \mathcal{G}_{1}} \mathrm{p}\left(\tilde{\mathbf{G}}_{1}\right) \mathrm{H}\left(\tilde{\mathbf{G}}_{1} \mathbf{x} \mid \mathbf{u}_{1}\right) .
$$

Suppose the proposition is true for $k-1$, then the proposition follows by successive application of the induction hypothesis, eqn. (11), and Prop. 5:

$$
\begin{aligned}
& \sum_{j=1}^{k} \frac{R_{j}}{j}=\frac{R_{k}}{k}+\sum_{j=1}^{k-1} \frac{R_{j}}{j} \\
\leq & \frac{R_{k}}{k}+\sum_{\tilde{\mathbf{G}}_{1} \in \mathcal{G}_{1}} \mathrm{p}\left(\tilde{\mathbf{G}}_{1}\right) \mathrm{H}\left(\tilde{\mathbf{G}}_{1} \mathbf{x}\right) \\
& -\frac{1}{k-1} \sum_{\tilde{\mathbf{G}}_{k-1} \in \mathcal{G}_{k-1}} \mathrm{p}\left(\tilde{\mathbf{G}}_{k-1}\right) \mathrm{H}\left(\tilde{\mathbf{G}}_{k-1} \mathbf{x} \mid \mathbf{u}_{k-1}\right) \\
\leq & \frac{1}{k} \sum_{\tilde{\mathbf{G}}_{k} \in \mathcal{G}_{k}} \mathrm{p}\left(\tilde{\mathbf{G}}_{k}\right)\left(\mathrm{H}\left(\tilde{\mathbf{G}}_{k} \mathbf{x} \mid \mathbf{u}_{k-1}\right)-\mathrm{H}\left(\tilde{\mathbf{G}}_{k} \mathbf{x} \mid \mathbf{u}_{k}\right)\right) \\
& +\sum_{\tilde{\mathbf{G}}_{1} \in \mathcal{G}_{1}} \mathrm{p}\left(\tilde{\mathbf{G}}_{1}\right) \mathrm{H}\left(\tilde{\mathbf{G}}_{1} \mathbf{x}\right) \\
& -\frac{1}{k-1} \sum_{\tilde{\mathbf{G}}_{k-1} \in \mathcal{G}_{k-1}} \mathrm{p}\left(\tilde{\mathbf{G}}_{k-1}\right) \mathbf{H}\left(\tilde{\mathbf{G}}_{k-1} \mathbf{x} \mid \mathbf{u}_{k-1}\right) \\
\leq & \frac{1}{k-1} \sum_{\tilde{\mathbf{G}}_{k-1} \in \mathcal{G}_{k-1}} \mathrm{p}\left(\tilde{\mathbf{G}}_{k-1}\right) \mathrm{H}\left(\tilde{\mathbf{G}}_{k-1} \mathbf{x} \mid \mathbf{u}_{k-1}\right) \\
& -\frac{1}{k} \sum_{\tilde{\mathbf{G}}_{k} \in \mathcal{G}_{k}} \mathrm{p}\left(\tilde{\mathbf{G}}_{k}\right) \mathrm{H}\left(\tilde{\mathbf{G}}_{k} \mathbf{x} \mid \mathbf{u}_{k}\right)+\sum_{\tilde{\mathbf{G}}_{1} \in \mathcal{G}_{1}} \mathrm{p}\left(\tilde{\mathbf{G}}_{1}\right) \mathrm{H}\left(\tilde{\mathbf{G}}_{1} \mathbf{x}\right) \\
& -\frac{1}{k-1} \sum_{\tilde{\mathbf{G}}_{k-1} \in \mathcal{G}_{k-1}} \mathrm{p}\left(\tilde{\mathbf{G}}_{k-1}\right) \mathbf{H}\left(\tilde{\mathbf{G}}_{k-1} \mathbf{x} \mid \mathbf{u}_{k-1}\right) \\
= & \sum_{\tilde{\mathbf{G}}_{1} \in \mathcal{G}_{1}} \mathrm{p}\left(\tilde{\mathbf{G}}_{1}\right) \mathbf{H}\left(\tilde{\mathbf{G}}_{1} \mathbf{x}\right)-\frac{1}{k} \sum_{\tilde{\mathbf{G}}_{k} \in \mathcal{G}_{k}} \mathrm{p}\left(\tilde{\mathbf{G}}_{k}\right) \mathrm{H}\left(\tilde{\mathbf{G}}_{k} \mathbf{x} \mid \mathbf{u}_{k}\right)
\end{aligned}
$$

This proves Prop. 6. The converse in Theorem 3 follows from Prop. 6 by setting $k=M$ and by noting that $\sum_{\tilde{\mathbf{G}}_{1} \in \mathcal{G}_{1}} \mathrm{p}\left(\tilde{\mathbf{G}}_{1}\right) \mathbf{H}\left(\tilde{\mathbf{G}}_{1} \mathbf{x}\right) \leq K$ and that $\mathbf{H}\left(\tilde{\mathbf{G}}_{M} \mathbf{x} \mid \mathbf{u}_{M}\right)=0$. The proof of achievability is exactly the same as the achievability proof found at the end of Appendix C.

\section{APPENDIX E}

Proof of Theorem 4: Structural Properties of Rate Delay TradeofF

The decoding deadline sequence $\mathbf{h}=(h(i), i=1, \ldots, N)$ is the minimum number of packets, say $j$, that must be received before the receiver is guaranteed to be able to decode the first $i$ frames. Thus $h:\{1, \ldots, N\} \rightarrow\{1, \ldots, M\}$ maps frame indices to packet reception counts (deadlines). The deadlines are by necessity non-decreasing: $h(i) \leq h(i+1)$. Because the delay metric $D_{p}(\mathbf{r})$ depends on $\mathbf{r}$ only through $g(i, \mathbf{r})$, we can break the optimization of $\mathbf{r}$ up into two coupled optimization problems, so that the optimal rate vector $\mathbf{r}^{*}$ is the solution to

$$
\rho\left(\mathbf{r}^{*}\right)=\max _{\mathbf{h} \mid \mathbb{E}[D(\mathbf{h})] \leq d} \max _{\mathbf{r} \in \mathcal{R} \mid g(i, \mathbf{r})=h(i), i=1, \ldots, N} \rho(\mathbf{r}) .
$$

We rearrange the definition of $g(i, \mathbf{r})(15)$ using the definition of $\rho(\mathbf{r})(13)$ as

$$
g(i, \mathbf{r})=\inf \left\{n \mid \frac{\sum_{j=1}^{n} R_{j}}{\sum_{j=1}^{M} R_{j}} \geq \frac{i}{N}\right\} .
$$

We can see that $\rho(\mathbf{r})$ depends only on the magnitude $\left(\|\cdot\|_{1}\right)$ of $\mathbf{r}$, while $g(i, \mathbf{r})$ depends only on the direction in which $\mathbf{r}$ points. The constraint (9), on the other hand, depends on both its magnitude and direction. This observation motivates a change of variables from $\mathbf{r}$ to $\gamma=\left(\gamma_{1}, \ldots, \gamma_{M}\right)$ and $\rho$ such that $\gamma_{j} \geq 0, \sum_{j=1}^{M} \gamma_{j}=1$ and $\rho=\rho(\mathbf{r})$. In particular, set

$$
\rho=\rho(\mathbf{r}), \quad \gamma_{j}=\frac{R_{j}}{\sum_{k=1}^{M} R_{k}}=\frac{R_{j}}{\rho N T_{s}}, j=1, \ldots, M .
$$

The constraints $\mathbf{r} \in \mathcal{R}$ and $g(i, \mathbf{r})=h(i)$ expressed in terms of $(\gamma, \rho)$ become:

$$
\begin{gathered}
\mathbf{r} \in \mathcal{R} \Leftrightarrow \sum_{j=1}^{M} \frac{\gamma_{j}}{j} \leq \frac{K}{N T_{s} \rho} \\
g(i, \mathbf{r})=h(i) \Leftrightarrow \inf \left\{n \mid \sum_{j=1}^{n} \gamma_{j} \geq \frac{i}{N}\right\}=h(i),
\end{gathered}
$$

for $i \in\{1, \ldots, N\}$. The inner maximization in (42) expressed in terms of $(\gamma, \rho)$ becomes:

$\max _{\boldsymbol{\gamma}, \boldsymbol{\rho}}\left\{\rho \mid \begin{array}{c}\sum_{j=1}^{M} \gamma_{j}=1, \boldsymbol{\gamma} \geq \mathbf{0}, \quad \sum_{j=1}^{M} \frac{\gamma_{j}}{j} \leq \frac{K}{N T_{s} \rho}, \\ \min \left\{n \mid \sum_{j=1}^{n} \gamma_{j} \geq \frac{i}{N}\right\}=h(i), i=1, \ldots, N\end{array}\right\}$.

For fixed $\gamma$, the optimal $\rho$ in (44) is

$$
\rho=\frac{K}{N T_{s}}\left(\sum_{j=1}^{M} \frac{\gamma_{j}}{j}\right)^{-1} .
$$

Note that we may undo the change of variables via

$$
R_{j}=\rho N T_{s} \gamma_{j}=K \frac{\gamma_{j}}{\sum_{k=1}^{M} \frac{\gamma_{k}}{k}}, j=1, \ldots, M
$$


Each decoding constraint can be decomposed into a pair of equations:

$$
\begin{gathered}
\inf \left\{n \mid \sum_{j=1}^{n} \gamma_{j} \geq \frac{i}{N}\right\}=h(i) \Leftrightarrow \\
\left(\sum_{j=1}^{h(i)-1} \gamma_{j}<\frac{i}{N} \text { and } \sum_{j=1}^{h(i)} \gamma_{j} \geq \frac{i}{N}\right),
\end{gathered}
$$

for all $i \in\{1, \ldots, N\}$. Applying this decomposition and substituting the optimal $\rho$ transforms the inner maximization in (42) to a minimization of $\sum_{j=1}^{M} \frac{\gamma_{j}}{j}$ :

$$
\min _{\gamma}\left\{\sum_{j=1}^{M} \frac{\gamma_{j}}{j} \mid \begin{array}{c}
\sum_{j=1}^{M} \gamma_{j}=1, \quad \gamma \geq \mathbf{0}, \quad \sum_{j=1}^{h(i)-1} \gamma_{j}<\frac{i}{N}, \\
\sum_{j=1}^{h(i)} \gamma_{j} \geq \frac{i}{N}, i=1, \ldots, N
\end{array}\right\} .
$$

In the absence of any decoding constraints, the objective $\sum_{j=1}^{M} \frac{\gamma_{j}}{j}$ is minimized over all non-negative $\gamma$ summing to one by setting $\gamma_{j}=0$ for $j=1, \ldots, M-1$, and $\gamma_{M}=1$. The optimal solution is thus to make each $\gamma_{j}$ as small as possible while obeying the decoding constraints $\mathbf{h}$. To handle these decoding constraints it is convenient to define several quantities associated with $\mathbf{h}$ :

$$
\begin{aligned}
& n=\#\{j: h(i)=j, i=1, \ldots, N\} \\
& \mathbf{l}=\left(l_{1}<l_{2}<\cdots<l_{n}\right)=\{j: h(i)=j, i=1, \ldots, N\} \\
& h^{-1}(j)=\max \{i: h(i)=j\}, j \in \mathbf{l} \\
& \mathbf{k}=\left(0=k_{0}<k_{1}<\cdots<k_{n}=N\right)=\left\{h^{-1}(j), j \in \mathbf{l}\right) \cup\{0\} \\
& \quad=\{i: h(i)<h(i+1), i=1, \ldots, N\} \cup\{0, N\} \\
& \boldsymbol{\Delta}=\left(\Delta_{1}=k_{1}-0, \Delta_{2}=k_{2}-k_{1}, \ldots, \Delta_{n}=N-k_{n-1}\right) .
\end{aligned}
$$

In words, $n$ is the number of distinct deadlines, $l$ is the vector of distinct deadlines, $h^{-1}(j)$ is the last frame index associated with each deadline $j \in \mathbf{l}, \mathbf{k}$ is the vector of last frame indices for each deadline augmented with $k_{0}=0$, and $\Delta$ is the vector of differences between the elements in $\mathbf{k}$. These quantities are illustrated in Fig. 7. Note that $n \leq \min \{M, N\}$ for all h. To minimize $\sum_{j=1}^{M} \frac{\gamma_{j}}{j}$ subject to decoding constraints, the optimal choice for $\gamma$ is to set

$$
\gamma_{h\left(k_{m}\right)}^{*}=\frac{k_{m}-k_{m-1}}{N}=\frac{\Delta_{m}}{N}, m=1, \ldots, n,
$$

and set all other $\gamma_{j}^{*}$ to zero. This assignment satisfies the decoding constraints while delaying assigning the mass in $\gamma$ to as high an index as possible. Note that this assignment satisfies the constraint that $\gamma \geq \mathbf{0}$ and the sum to one constraint:

$$
\sum_{j=1}^{M} \gamma_{j}^{*}=\sum_{m=1}^{n} \gamma_{h\left(k_{m}\right)}^{*}=\sum_{m=1}^{n} \frac{k_{m}-k_{m-1}}{N}=\frac{k_{n}-k_{0}}{N}=1 .
$$

Further, the objective in (47) becomes:

$$
\sum_{j=1}^{M} \frac{\gamma_{j}^{*}}{j}=\sum_{m=1}^{n} \frac{\gamma_{h\left(k_{m}\right)}^{*}}{h\left(k_{m}\right)}=\frac{1}{N} \sum_{m=1}^{n} \frac{\Delta_{m}}{l_{m}}=\frac{1}{N} \sum_{m=1}^{n(\mathbf{h})} \frac{\Delta(\mathbf{h})_{m}}{l(\mathbf{h})_{m}},
$$

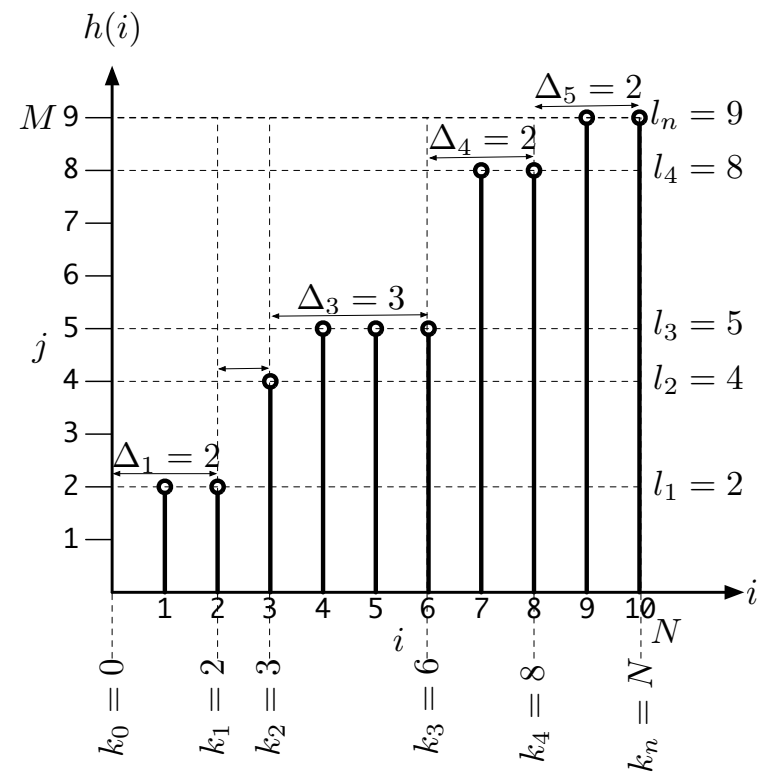

Fig. 7. Illustration of the decoding deadline sequence $\mathbf{h}=$ $(h(1), \ldots, h(N))$, where $h(i)$ is the number of packets that must be received to guarantee that frame $i$ may be decoded for the case $N=10$ frames and $M=9$ packets. Using the notation $n, \mathbf{l}, \mathbf{k}, \Delta$ introduced the proof of the theorem, there are $n=5$ distinct deadlines, namely $\mathbf{l}=\left(l_{1}, \ldots, l_{n}\right)=$ $(2,4,5,8,9)$, and the last frame indices associated with each deadline are $\mathbf{k}=\left(k_{0}, \ldots, k_{n}\right)=(0,2,3,6,8,10)$. The duration of each deadline is given as $\Delta=\left(\Delta_{1}, \ldots, \Delta_{n}\right)=(2,1,3,2,2)$.

where the last expression makes explicit that $n, \Delta_{m}, l_{m}$ are each computed from the decoding deadlines $\mathbf{h}$. The associated maximum sum rate under $\mathbf{h}$ is then

$$
\rho(\mathbf{h})=\frac{K}{N T_{s}}\left(\sum_{j=1}^{M} \frac{\gamma_{j}^{*}}{j}\right)^{-1}=\frac{K}{T_{s}}\left(\sum_{m=1}^{n(\mathbf{h})} \frac{\Delta(\mathbf{h})_{m}}{l(\mathbf{h})_{m}}\right)^{-1} .
$$

Finally, the rate delay tradeoff (16) expressed in terms of the decoding deadlines is

$$
\max _{\mathbf{h}: \mathbb{E}\left[D_{p}(\mathbf{h})\right] \leq d} \frac{K}{T_{s}}\left(\sum_{m=1}^{n(\mathbf{h})} \frac{\Delta(\mathbf{h})_{m}}{l(\mathbf{h})_{m}}\right)^{-1} .
$$

The remaining optimization is solved by exhaustive enumeration over all decoding constraints that satisfy the delay constraint $d$. We have thus shown that the rate delay tradeoff, although correctly posed as a continuous optimization, can be found as the solution to an integer programming problem in the decoding deadlines $\mathbf{h}$ in the uniform case.

\section{APPENDIX F \\ Proof of THEOREM 6: CONSTANT INTERARrival TIME Approximate Rate Delay TradeofF}

The calculus of variations problem (21) for normalized delay bound $\bar{d} \in[0,1]$ and $p=1$ in (19) may be written as

$$
\frac{1}{\bar{\rho}_{\bar{d}}^{*}}=\inf _{\mathbf{z} \in \mathcal{Z}(\bar{d})} \int_{0}^{1} \frac{1}{\mathbf{z}(t)} \mathrm{d} t .
$$


where $\mathcal{Z}(\bar{d})$ is the set of differentiable $\mathrm{z}:[0,1] \rightarrow[0,1]$ obeying

$$
\int_{0}^{1}(\mathrm{z}(t)-t)^{+} \mathrm{d} t \leq \frac{M-1}{2 M} \bar{d}, \frac{\partial \mathrm{z}(t)}{\partial t} \geq 0 .
$$

Note that the objective function is monotone decreasing in $\mathrm{z}(t)$, and if $\mathrm{z}(t)<t$, the delay does not change by changing it to $\mathrm{z}(t)=t$. This allows:

$$
\frac{1}{\bar{\rho}_{\bar{d}}^{*}}=\min _{\mathbf{z} \in \mathcal{Z}(\bar{d}), z(t) \geq t} \int_{0}^{1} \frac{1}{\mathrm{z}(t)} \mathrm{d} t .
$$

The Lagrangian is

$$
\begin{aligned}
\mathcal{L}(\mathrm{z}, \mu, \mathrm{a}, \mathrm{b})= & \int_{0}^{1} \frac{1}{\mathrm{z}(t)} \mathrm{d} t-\mu\left(\int_{0}^{1}(\mathrm{z}(t)-t) \mathrm{d} t-\frac{M-1}{2 M} \bar{d}\right) \\
& +\int_{0}^{1} \mathrm{a}(t) \frac{\partial \mathrm{z}(t)}{\partial t} \mathrm{~d} t+\int_{0}^{1} \mathrm{~b}(t)(\mathrm{z}(t)-t) \mathrm{d} t,(53)
\end{aligned}
$$

where $\mathrm{a}(t)$ is the Lagrange multiplier on the constraint $\frac{\partial z(t)}{\partial t} \geq$ 0 at $t$ and $\mathrm{b}(t)$ is the multiplier on the constraint $\mathrm{z}(t) \geq t$ at $t$, for each $t \in[0,1]$. The solution to the variational problem (53) must satisfy the algebraic equation, obtained by differentiating (53) with respect to $z(t)$ at each $t=t_{0}$ :

$$
-\frac{1}{\left(\mathbf{z}^{*}\left(t_{0}\right)\right)^{2}}-\mu+\alpha\left(t_{0}\right) \mathbf{1}_{\left\{\frac{\partial z^{*}\left(t_{0}\right)}{\partial t}\right\}}+\beta\left(t_{0}\right) \mathbf{1}_{\left\{z\left(t_{0}\right)=t_{0}\right\}}=0,
$$

for $t_{0} \in[0,1]$. Here $\alpha\left(t_{0}\right)$ and $\beta\left(t_{0}\right)$ are defined to be non-zero when the slope of $\mathrm{z}\left(t_{0}\right)$ is zero or $\mathrm{z}\left(t_{0}\right)=t_{0}$, respectively. These constraints dictate the values of $\alpha\left(t_{0}\right)$ and $\beta\left(t_{0}\right)$, although it is not necessary to solve for them explicitly. Solving this for $z^{*}$, we see that a necessary condition for $z^{*}$ to be an extremal for the constrained optimization is

$$
\mathrm{z}^{*}\left(t_{0}\right)=\left(\mu+\alpha\left(t_{0}\right) \mathbf{1}_{\left\{\frac{\partial z^{*}\left(t_{0}\right)}{\partial t}=0\right\}}+\beta\left(t_{0}\right) \mathbf{1}_{\left\{\mathrm{z}^{*}\left(t_{0}\right)=t_{0}\right\}}\right)^{-\frac{1}{2}},
$$

for each $t_{0} \in[0,1]$. From this equation it follows that $z^{*}$ will be a piecewise function that is either constant or increasing at unit slope, i.e., $\mathrm{z}(t)=t$. Furthermore, the inverse rate objective function is decreased more by having the constant portion of the function for small $t$. Thus the optimal $z^{*}$ takes the form

$$
\mathrm{z}^{*}(t):=\left\{\begin{array}{l}
\zeta, 0 \leq t \leq \zeta \\
t \quad \zeta<t \leq 1
\end{array}\right.
$$

for some $\zeta \in[0,1]$. This gives a normalized rate of

$$
\bar{\rho}=\left(\int_{0}^{1} \frac{1}{\mathrm{z}^{*}(t)} \mathrm{d} t\right)^{-1}=(1-\log (\zeta))^{-1}
$$

and a normalized delay of

$$
\bar{\delta}=\frac{2 M}{M-1}\left(\zeta^{2}-\frac{\zeta^{2}}{2}\right)=\frac{M \zeta^{2}}{M-1} .
$$

Since $\zeta=\sqrt{\frac{M-1}{M} \bar{\delta}}$, the approximate (asymptotically accurate) rate delay tradeoff for delay bound $\bar{d}$ and rate bound $\bar{\rho}$ is:

$$
\begin{aligned}
& \bar{\rho}_{\bar{d}}^{*}=\left(1-\log \left(\sqrt{\frac{M-1}{M} \bar{d}}\right)\right)^{-1}, \\
& \bar{\delta}_{\bar{\rho}}^{*}=\frac{M}{M-1} \exp \left(2-\frac{2}{\bar{\rho}}\right) .
\end{aligned}
$$

\section{APPENDIX G}

Proof of THEOREM 7: I.I.D. EXPONENTIAL ARRIVAL Time Approximate Rate Delay TradeofF

The fundamental rate delay tradeoff (21) for i.i.d. exponential arrival times with parameter $\lambda$ and with approximate normalized delay functional (27) is:

$$
\min _{\mathbf{z} \in \mathcal{Z}^{\prime}(\bar{d})} \int_{0}^{1} \frac{1}{\mathbf{z}(t)} \mathrm{d} t,
$$

where $\mathcal{Z}^{\prime}(\bar{d})$ is the set of differentiable $\mathrm{z}:[0,1] \rightarrow[0,1]$ obeying

$$
\begin{array}{r}
\int_{0}^{1}\left(-\lambda^{-1} \log (1-\mathrm{z}(t))-t N T_{s}\right)^{+} \mathrm{d} t \leq c(M) \bar{d} \\
\frac{\partial \mathbf{z}(t)}{\partial t} \geq 0, \text { and } c(M)=\int_{0}^{1} \mathbb{E}\left[\left(\tau_{M}-N T_{s} t\right)^{+}\right] \mathrm{d} t
\end{array}
$$

is the denominator in (19) (for $p=1$ ) and is independent of the functional $z$. Since the inverse rate (which we want to minimize) is monotone decreasing in $\mathrm{z}(t)$ for all $t \in[0,1]$, and the delay is insensitive to any increase in $z(t)$ when $-\lambda^{-1} \log (1-z(t)) \geq N T_{s} t$, it suffices to consider only those $\mathrm{z}$ such that $\mathrm{z}(t) \geq 1-\mathrm{e}^{-\lambda N T_{s} t}$. We parameterize this set of $\mathrm{z}$ as $\mathrm{z}(t)=1-\mathrm{e}^{-\lambda N T_{s} t-\mathrm{y}(t)}$ with $\mathrm{y}(t) \geq 0$, so that

$$
\begin{aligned}
\frac{\partial \mathrm{z}(t)}{\partial t} \geq 0 \Leftrightarrow\left(\lambda N T_{s}+\frac{\partial \mathrm{y}(t)}{\partial t}\right) \mathrm{e}^{-\lambda N T_{s} t-\mathrm{y}(t)} & \geq 0 \Leftrightarrow \\
\frac{\partial \mathrm{y}(t)}{\partial t} & \geq-\lambda N T_{s} .
\end{aligned}
$$

This allows us to express (56) in terms of the functional y:

$$
\inf _{\mathrm{y} \in \mathcal{Y}(\bar{d})} \int_{0}^{1} \frac{1}{1-\mathrm{e}^{-\lambda N T_{s} t-\mathrm{y}(t)}} \mathrm{d} t
$$

where $\mathcal{Y}(\bar{d})$ is the set of differentiable $\mathrm{y}:[0,1] \rightarrow[0, \infty)$ obeying

$$
\int_{0}^{1} \mathrm{y}(t) \mathrm{d} t \leq \lambda c(M) \bar{d}, \frac{\partial \mathrm{y}(t)}{\partial t} \geq-\lambda N T_{s}, \mathrm{y}(t) \geq 0 .
$$

The Lagrangian $\mathcal{L}(\mathrm{y}, \mu, \mathrm{a}, \mathrm{b})$ is

$$
\begin{array}{r}
\int_{0}^{1} \frac{1}{1-\mathrm{e}^{-\lambda N T_{s} t-\mathrm{y}(t)} \mathrm{d} t-\mu\left(\int_{0}^{1} \mathrm{y}(t) \mathrm{d} t-\lambda c(M) \bar{d}\right)+} \\
\int_{0}^{1} \mathrm{a}(t)\left(\frac{\partial \mathrm{y}(t)}{\partial t}+\lambda N T_{s}\right) \mathrm{d} t+\int_{0}^{1} \mathrm{~b}(t) \mathrm{y}(t) \mathrm{d}(59)
\end{array}
$$

where $\mathrm{a}(t)$ is the Lagrange multiplier on the constraint $\frac{\partial \mathrm{y}(t)}{\partial t} \geq$ 0 at $t$ and $\mathbf{b}(t)$ is the multiplier on the constraint $\mathrm{y}(t) \geq 0$ at $t$, for each $t \in[0,1]$. The solution to the variational problem (58) must satisfy the algebraic equation, obtained by differentiating (59) with respect to $\mathrm{y}(t)$ at each $t=t_{0}$ :

$$
\begin{aligned}
-\frac{\mathrm{e}^{-\lambda N T_{s} t_{0}-\mathrm{y}^{*}\left(t_{0}\right)}}{\left(1-\mathrm{e}^{-\lambda N T_{s} t_{0}-\mathrm{y}^{*}\left(t_{0}\right)}\right)^{2}}-\mu+ & \alpha\left(t_{0}\right) \mathbf{1}_{\left\{\frac{\partial \mathrm{y}^{*}\left(t_{0}\right)}{\partial t}=-\lambda N T_{s}\right\}} \\
& \left.+\beta\left(t_{0}\right) \mathbf{1}_{\left\{\mathrm{y}^{*}\left(t_{0}\right)=0\right\}}=0,60\right)
\end{aligned}
$$

for $t_{0} \in[0,1]$. Here $\alpha\left(t_{0}\right)$ and $\beta\left(t_{0}\right)$ are defined to be non-zero when the slope of $\mathrm{y}\left(t_{0}\right)$ is $-\lambda N T_{s}$ or $\mathrm{y}\left(t_{0}\right)=0$, respectively. These constraints dictate the values of $\alpha\left(t_{0}\right)$ and $\beta\left(t_{0}\right)$, although it is not necessary to solve for them explicitly. 
From this equation it follows that $\mathrm{y}^{*}$ will be a piecewise function that is either decreasing at slope $-\lambda N T_{s}$ or is equal to zero. To see this, note that if both inequality constraints $\frac{\partial \mathrm{y}(t)}{\partial t} \geq-\lambda N T_{s}$ and $\mathrm{y}(t) \geq 0$ are inactive for some interval $t \in\left(t_{r}, t_{l}\right)$, so that the only non-zero Lagrange multiplier is $\mu \leq 0$, we find that $\mathrm{z}(t)$ must be a constant on this interval, since (60) in this case could be rewritten as

$$
\frac{1-\mathrm{z}(t)}{(\mathrm{z}(t))^{2}}=-\mu, \quad t \in\left(t_{r}, t_{l}\right)
$$

but this implies $\frac{\partial \mathrm{y}(t)}{\partial t}=-\lambda N T_{s}$, which is a contradiction because this constraint was assumed inactive. Thus, one of the two constraints $\frac{\partial \mathrm{y}(t)}{\partial t} \geq-\lambda N T_{s}$ and $\mathrm{y}(t) \geq 0$ is always active, and this dictates the form of $\mathrm{y}(t)$. Thus, the optimal $\mathrm{y}^{*}$ takes the form

$$
\mathrm{y}^{*}(t)=\left\{\begin{array}{ll}
\lambda N T_{s}(\zeta-t), & 0 \leq t \leq \zeta \\
0, & \zeta<t \leq 1
\end{array},\right.
$$

for some $\zeta \in[0,1]$. The delay constraint dictates the optimal value of $\zeta$ :

$$
\begin{aligned}
\int_{0}^{1} \mathrm{y}^{*}(t) \mathrm{d} t=\lambda c(M) \bar{d} \Rightarrow \frac{1}{2} \lambda N T_{s}\left(\zeta^{*}\right)^{2} & =\lambda c(M) \bar{d} \Rightarrow \\
\zeta^{*} & =\sqrt{\frac{2 c(M) \bar{d}}{N T_{s}}} .
\end{aligned}
$$

Further, the optimal $z^{*}$ takes the form

$$
\mathrm{z}^{*}(t)=\left\{\begin{array}{ll}
1-\mathrm{e}^{-\lambda N T_{s} \zeta^{*}}, & 0 \leq t \leq \zeta^{*} \\
1-\mathrm{e}^{-\lambda N T_{s} t}, & \zeta^{*}<t \leq 1
\end{array} .\right.
$$

The corresponding inverse normalized rate is

$$
\begin{aligned}
& \frac{1}{\bar{\rho}_{\bar{d}}^{*}}=\int_{0}^{1} \frac{1}{\mathrm{z}^{*}(t)} \mathrm{d} t \\
= & \int_{0}^{\zeta^{*}} \frac{1}{1-\exp \left(-\lambda N T_{s} \zeta^{*}\right)} \mathrm{d} t+\int_{\zeta^{*}}^{1} \frac{1}{1-\exp \left(-\lambda N T_{s} t\right)} \mathrm{d} t \\
= & \frac{\zeta^{*}}{1-\exp \left(-\lambda N T_{s} \zeta^{*}\right)}+\frac{1}{\lambda N T_{s}} \log \left(\frac{\exp \left(\lambda N T_{s}\right)-1}{\exp \left(\lambda N T_{s} \zeta^{*}\right)-1}\right) \\
= & \frac{\sqrt{2 c(M) \bar{d}}}{\sqrt{N T_{s}}\left(1-\exp \left(-\lambda \sqrt{2 N T_{s} c(M) \bar{d}}\right)\right)} \\
& +\frac{1}{\lambda N T_{s}} \log \left(\frac{\exp \left(\lambda N T_{s}\right)-1}{\exp \left(\lambda \sqrt{2 N T_{s} c(M) \bar{d}}\right)-1}\right) .
\end{aligned}
$$

It remains to approximate $c(M)$. To do this, we first find the limiting distribution of a normalization of $\tau_{M}$ to be a Gumbel distribution. In the limit, $\tau_{M}$ is increasingly concentrated around its mean because its variance does not scale with $M$ while its mean does. This allows us to approximate the delay by replacing $\tau_{M}$ by its mean for very large $M$. In more detail, following [47] pp. 299-300, we find that $\tilde{\tau}_{M}=\lambda \tau_{M}-\log (M)$ has a Gumbel distribution with cumulative distribution function $F(t)=\exp \left(-\mathrm{e}^{-t}\right)$. Because the mean of this distribution is the Euler Mascheroni constant $\mathbb{E}\left[\tilde{\tau}_{M}\right] \rightarrow \gamma \approx 0.5772156649$ as $M \rightarrow \infty$, it follows that $\mathbb{E}\left[\tau_{M}\right] \approx \frac{\gamma+\log M}{\lambda}$ for large $M$. The asymptotic variances are $\operatorname{Var}\left(\tilde{\tau}_{M}\right) \rightarrow \frac{\pi^{2}}{6}$ and thus $\operatorname{Var}\left(\tau_{M}\right) \rightarrow \frac{\pi^{2}}{6 \lambda^{2}}$. The fact that the mean of $\tau_{M}$ grows unbounded in $M$ while the asymptotic variance of $\tau_{M}$ is independent of $M$ allows us to approximate the expectation

$$
\begin{aligned}
\mathbb{E}\left[\left(\tau_{M}-N T_{s} t\right)^{+}\right] & \approx\left(\mathbb{E}\left[\tau_{M}\right]-N T_{s} t\right)^{+} \\
& \approx\left(\frac{\gamma+\log M}{\lambda}-N T_{s} t\right)^{+} .
\end{aligned}
$$

Integrating this over $t$ yields

$$
\begin{aligned}
c(M) & \approx \int_{0}^{1}\left(\frac{\gamma+\log M}{\lambda}-N T_{s} t\right)^{+} \mathrm{d} t \\
& =\int_{0}^{\min \left\{\frac{\gamma+\log M}{\lambda N T_{s}}, 1\right\}}\left(\frac{\gamma+\log M}{\lambda}-N T_{s} t\right) \mathrm{d} t \\
& =\left\{\begin{array}{cl}
\frac{(\gamma+\log M)^{2}}{2 \lambda^{2} N T_{s}} & N>\frac{\gamma+\log M}{\lambda T_{s}} \\
\frac{\gamma+\log M}{\lambda}-\frac{N T_{s}}{2} & N \leq \frac{\gamma+\log M}{\lambda T_{s}}
\end{array}\right.
\end{aligned}
$$

We may then substitute this into (63) to obtain the rate delay tradeoff for large $M$ given by

$$
\bar{\rho}_{\bar{d}}^{*} \approx \lambda N T_{s}\left(\frac{(\gamma+\log M) \sqrt{\bar{d}}}{1-\mathrm{e}^{-\gamma \sqrt{d}} M^{-\sqrt{\bar{d}}}}+\log \frac{\mathrm{e}^{\lambda N T_{s}}-1}{\mathrm{e}^{\gamma \sqrt{d}} M^{\sqrt{\bar{d}}}-1}\right)^{-1}
$$

when $N>\frac{\gamma+\log M}{\lambda T_{s}}$ and

$$
\begin{aligned}
\bar{\rho}_{\bar{d}}^{*} \approx & \lambda N T_{s}\left[\frac{\sqrt{\left(2 \gamma+2 \log M-\lambda N T_{s}\right) \lambda N T_{s} \bar{d}}}{1-\exp \left(-\sqrt{\left(2 \gamma+2 \log M-\lambda N T_{s}\right) \lambda N T_{s} \bar{d}}\right)}\right. \\
& \left.+\log \frac{\exp \left(\lambda N T_{s}\right)-1}{\exp \left(\sqrt{\left(2 \gamma+2 \log M-\lambda N T_{s}\right) \lambda N T_{s} \bar{d}}\right)-1}\right]^{-1}
\end{aligned}
$$

when $N \leq \frac{\gamma+\log M}{\lambda T_{s}}$.

\section{REFERENCES}

[1] J. Walsh and S. Weber, "Coding to reduce delay on a permutation channel," in 45th Allerton Conference on Communication, Control, and Computing, Sept. 2007.

[2] _ "A concatenated network coding scheme for multimedia transmission," in Fourth Workshop on Network Coding, Theory, and Applications (NetCod 2008), Jan. 2008, pp. 91-96.

[3] J. M. Walsh, S. Weber, and C. Maina, "Optimal Rate Delay Tradeoffs for Multipath Routed and Network Coded Networks," in IEEE International Symposium on Information Theory (ISIT), 2008.

[4] D. S. Lun, "Efficient operation of coded packet networks," Ph.D. dissertation, Massachusetts Institute of Technology, 2006.

[5] T. Ho, M. Médard, R. Koetter, D. R. Karger, M. Effros, J. Shi, and B. Leong, "A random linear network coding approach to multicast," IEEE Transactions on Information Theory, vol. 52, no. 10, pp. 44134430, October 2006.

[6] C. Hopps, "Analysis of an Equal-Cost Multi-Path Algorithm," Nov. 2000, IETF Network Working Group RFC 2992. [Online]. Available: http://tools.ietf.org/html/rfc2992

[7] D. Thaler and C. Hopps, "Multipath Issues in Unicast and Multicast Next-Hop Selection," Nov. 2000, IETF Network Working Group RFC 2991. [Online]. Available: http://tools.ietf.org/html/rfc2991

[8] T. M. Cover, "Comments on broadcast channels," IEEE Trans. Inform. Theory, vol. IT-44, no. 6, pp. 2524-2530, October 1998.

[9] — - "Broadcast channels," IEEE Trans. Inform. Theory, vol. IT-18, no. 1, pp. 2-14, January 1972.

[10] P. P. Bergmans, "Random coding theorem for broadcast channels with degraded components," IEEE Trans. Inform. Theory, vol. IT-19, no. 2, pp. 197-207, March 1973.

[11] A. Albanese, J. Blömer, J. Edmonds, M. Luby, and M. Sudan, "Priority encoded transmission," IEEE Trans. Inform. Theory, vol. 42, no. 6, pp. 1737-1744, Nov. 1996.

[12] D. Silva and F. R. Kschischang, "Rank-metric codes for priority encoding transmission with network coding," in Canadian Workshop on Information Theory, Edmonton, AB, Canada, June 2007, pp. 81-84. 
[13] R. Ahlswede, N. Cai, S.-Y. R. Li, and R. W. Yeung, "Network information flow," IEEE Trans. Inform. Theory, vol. 46, no. 4, pp. 1204-1216, July 2000.

[14] R. W. Yeung, S.-Y. R. Li, N. Cai, and Z. Zhang, "Network coding theory," Foundation and Trends in Communications and Information Theory, vol. 2, no. 4-5, pp. $241-381,2005$.

[15] C. Fragouli, J.-Y. Le Boudec, and J. Widmer, "Network coding: An instant primer," ACM SIGCOMM Computer Communication Review, vol. 36, no. 1, pp. 63-68, 2006.

[16] Z. Zhang, "Some recent progresses in network error correction coding theory," in Fourth Workshop on Network Coding, Theory, and Applications (NetCod 2008), Hong Kong, China, Jan. 2008.

[17] D. Silva, F. R. Kschischang, and R. Koetter, "A rank-metric approach to error control in random network coding," in 2007 IEEE Information Theory Workshop on Information Theory for Wireless Networks, July 2007, pp. 1-5.

[18] R. Koetter and F. R. Kschischang, "Coding for errors and erasures in random network coding," in IEEE International Symposium on Information Theory (ISIT), Nice, France, June 2007.

[19] D. Silva, F. R. Kschischang, and R. Koetter, "A Rank-Metric Approach to Error Control in Random Network Coding," IEEE Trans. Inform. Theory, vol. 54, no. 9, pp. 3951-3967, Sept. 2008.

[20] P. A. Chou, Y.Wu, and K. Jain, "Practical network coding," in 41st Annual Allerton Conf. on Communication, Control, and Computing, Monticello, IL, Sept. 2003.

[21] D. S. Lun, N. Ratnakar, M. Médard, R. Koetter, D. R. Karger, T. Ho, E. Ahmed, and F. Zhao, "Minimum-cost multicast over coded packet networks," IEEE Transactions on Information Theory, vol. 52, no. 6, pp. 2608-2623, June 2006.

[22] L. Keller, E. Drinea, and C. Fragouli, "Online broadcasting with network coding," in Fourth Workshop on Network Coding, Theory, and Applications (NetCod 2008), Hong Kong, China, Jan. 2008.

[23] J. Byers, M. Luby, M. Mitzenmacher, and A. Rege, "A digital fountain approach to reliable distribution of bulk data," in ACM SIGCOMM '98, Vancouver, BC, Canada, Jan. 1998, p. 5667.

[24] A. Shokrollahi, "Raptor codes," IEEE Trans. Inform. Theory, vol. 52, no. 6, pp. 2551-2567, 2006.

[25] R. G. Gallager, Low Density Parity-Check Codes. Cambridge, MA: MIT Press, 1963.

[26] _ "Low-density parity-check codes." IRE Trans. Information Theory, vol. 2, pp. 21-28, 1962

[27] T. J. Richardson, M. A. Shokrollahi, and R. L. Urbanke, "Design of capacity-approaching irregular low-density parity-check codes," IEEE Trans. Inform. Theory, no. 2, pp. 619-639, Feb. 2001.

[28] M. Luby, M. Mitzenmacher, A. Shokrollahi, and D. Spielman, "Improved low-density parity-check codes using irregular graphs," IEEE Trans. Inform. Theory, vol. 47, no. 2, pp. 585-598, Feb. 2001.

[29] D. J. C. MacKay, "Good error-correcting codes based on very sparse matrices." IEEE Trans. Inform. Theory, vol. 45, pp. 399-431, Mar. 1999.

[30] S. Sanghavi, "Intermediate performance of rateless codes," in Information Theory Workshop (ITW), Sept. 2007, pp. 478-482.

[31] A. Ashikhmin, G. Kramer, and S. ten Brink, "Extrinsic information transfer functions: model and erasure channel properties," IEEE Trans. Inform. Theory, vol. 50, no. 11, pp. 2657-2673, Nov. 2004.

[32] C. Berrou, A. Glavieux, and P. Thitimajshima, "Near Shannon limit error-correcting coding and decoding: Turbo-codes." in ICC 93, vol. 2, Geneva, May 1993, pp. 1064-1070.

[33] C. Berrou and A. Glavieux, "Near optimum error correction coding and decoding: Turbo codes.” IEEE Trans. Commun., vol. 44, pp. 1262-1271, Oct. 1996.

[34] S. Benedetto and G. Montorsi, "Unveiling turbo codes: Some results on parallel concatenated coding structures." IEEE Trans. Inform. Theory, vol. 42, pp. 409-428, Mar. 1996.

[35] S. Benedetto, D. Divsalar, G. Montorsi, and F. Pollara, "Serial concatenation of interleaved codes: performance analysis, design, and iterative decoding," IEEE Trans. Inform. Theory, vol. 44, pp. 909-926, May 1998.

[36] S. Boucheron and M. R. Slamatian, "About priority encoding transmission," IEEE Trans. Inform. Theory, vol. 46, no. 2, pp. 699-705, Mar. 2000.

[37] R. L. Urbanke and A. D. Wyner, "Methods and apparatus for packetizing data for transmission through an erasure broadcast channel," U.S. Patent $6175944,1997$.

[38] - "Packetizing for the erasure broadcast channel with an internet application," 1997, preprint. [Online]. Available: citeseer.ist.psu.edu/urbanke97packetizing.html
[39] C. Huang, R. Janakiraman, and L. Xu, "Loss-resilient on-demand media streaming using priority encoding," in Proc. ACM Multimedia (MM 2004), New York, NY, Oct. 2004, pp. 152-159.

[40] R. Janakiraman and L. Xu, "Layered priority encoded transmission for video streaming to heterogeneous clients," in Proceedings of the 2004 IEEE International Symposium on Information Theory (IEEE ISIT 2004), Chicago, IL, July 2004, p. 331.

[41] — , "Efficient and flexible parallel retrieval using priority encoded transmission," in Proceedings of the 14th ACM International Workshop on Network and Operating Systems Support for Digital Audio and Video (ACM NOSSDAV 2004), Kinsale, Ireland, June 2004, pp. 48-53.

[42] R. G. Gallager, Information Theory and Reliable Communication. J. Wiley \& Sons, 1968.

[43] R. Berry and R. Gallager, "Communication over fading channels with delay constraints," IEEE Trans. Inform. Theory, vol. 48, no. 5, pp. 1135 $-1149,2002$

[44] R. G. Gallager, "Capacity and coding for degraded broadcast channels," Probl. Pered. Inform., vol. 10, no. 3, pp. 3-14, 1974.

[45] F. Y. Wan, Introduction to the Calculus of Variations and Its Applications. Chapman and Hall Mathematics, 1995.

[46] I. Gelfand and S. Fomin, Calculus of Variations. Dover, 1963.

[47] H. David and H. Nagaraja, Order Statistics. Hoboken, N.J.: John Wiley \& Sons, 2003.

[48] Shu Lin and D. J. Costello, Jr., Error Control Coding. Pearson Prentice Hall, 2004.

[49] A. E. Gamal, "The feedback capacity of degraded broadcast channels," IEEE Trans. Inform. Theory, vol. IT-24, no. 3, pp. 379-381, May 1978.

[50] T. M. Cover and J. A. Thomas, Elements of Information Theory, 2nd. Ed. John Wiley and Sons, 2006.

[51] T. S. Han, "Nonnegative entropy measures of multivariate symmetric correlations," Inf. Control, vol. 36, no. 2, pp. 133-156, 1978.

John MacLaren Walsh (S 2001, M 2007) was born in Carbondale, IL in 1981. He received the B.S. (magna cum laude), M.S., and Ph.D. from Cornell University, Ithaca, NY in 2002, 2004, and 2006 respectively.

In September, 2006 he joined the Department of Electrical and Computer Engineering at Drexel University, Philadelphia, PA where he is currently an assistant professor. He is a member of HKN and TBP. His current research interests include: (a) delay mitigating codes and rate delay tradeoffs in multipath routed and network coded networks, (b) joint source separation and identification, and (c) the performance and convergence of distributed collaborative estimation in wireless sensor networks via expectation propagation.

Steven Weber (S 1991, M 1997) was born in Milwaukee, WI and received his B.S. degree in 1996 from Marquette University in Milwaukee, WI, and his M.S. and Ph.D. degrees from The University of Texas at Austin in 1999 and 2003 respectively.

He joined the Department of Electrical and Computer Engineering at Drexel University in Philadelphia, PA in 2003 where he is currently an Associate Professor. His research interests are centered around mathematical modeling of computer and communication networks, with much of his recent work focused on wireless ad hoc networks.

Ciira wa Maina (S 2008) was born in Nairobi, Kenya in 1983 . He received the B.S. degree from the University of Nairobi, Kenya in 2007 and is currently working towards the Ph.D. degree at Drexel University, Philadelphia, PA, USA. His research interests include approximate Bayesian inference techniques applied to joint sound source separation and identification and the performance and convergence of blind adaptive channel shorteners. 